\title{
Spin Chemical Control of Photoinduced Electron-Transfer Processes in Ruthenium(II)-Trisbipyridine-Based Supramolecular Triads: 2. The Effect of Oxygen, Sulfur, and Selenium as Heteroatom in the Azine Donor
}

\author{
Matthew T. Rawls, ${ }^{\dagger}$ Georg Kollmannsberger, ${ }^{\dagger}$ C. Michael Elliott, ${ }^{*}, \dagger$ and Ulrich E. Steiner $*$, \\ Department of Chemistry, Colorado State University, Fort Collins, Colorado 80523, and Fachbereich Chemie, \\ Universität Konstanz, Konstanz, Germany, D-78457
}

\begin{abstract}
Nanosecond time-resolved absorption studies in a magnetic field ranging from 0 to $2.0 \mathrm{~T}$ have been performed on a series of covalently linked donor(PXZ)-Ru(bipyridine) $)_{3}-$ acceptor(diquat) complexes $\left(\mathrm{D}-\mathrm{C}^{2+}-\mathrm{A}^{2+}\right)$. In the PXZ moiety, the heteroatom ( $\mathrm{X}=\mathrm{O}$ (oxygen), $\mathrm{T}$ (sulfur), and $\mathrm{S}$ (selenium)) is systematically varied to study spin-orbit coupling effects. On the nanosecond time scale, the first detectable photoinduced electrontransfer product after exciting the chromophore $\mathrm{C}^{2+}$ is the charge-separated (CS) state, $\mathrm{D}^{+}-\mathrm{C}^{2+}-\mathrm{A}^{+}$, where an electron of the PXZ moiety, D, has been transferred to the diquat moiety, $\mathrm{A}^{2+}$. The magnetic-field-dependent kinetic behavior of charge recombination (monoexponential at $0 \mathrm{~T}$ progressing to biexponential for all three complexes with increasing field) can be quantitatively modeled by the radical pair relaxation mechanism assuming creation of the CS state with pure triplet spin correlation $\left({ }^{3} \mathrm{CS}\right)$. Magnetic-field-independent contributions to the rate constant $k_{\mathrm{r}}$ of $\mathrm{T}_{ \pm} \rightarrow\left(\mathrm{T}_{0}, \mathrm{~S}\right)$ relaxation are about $4.5 \times 10^{5} \mathrm{~s}^{-1}$ for DCA-POZ and -PTZ (due to a vibrational mechanism) and $3.5 \times 10^{6} \mathrm{~s}^{-1}$ for DCA-PSZ (due to spin rotational mechanism). Recombination to the singlet ground state is allowed only from the ${ }^{1} \mathrm{CS}$ spin level; spin-forbidden recombination from ${ }^{3} \mathrm{CS}$ seems negligible even for DCA-PSZ. The field dependence of $k_{\mathrm{r}}$ (field-dependent recombination) can be decomposed into the contributions of various relaxation mechanisms. For all compounds, the electron spin dipolar coupling relaxation mechanism dominates the field dependence of $\tau_{\text {slow }}$ at fields up to about 100 mT. Spin relaxation due to the $g$-tensor anisotropy relaxation mechanism accounts for the field dependence of $\tau_{\text {slow }}$ for DCA-PSZ at high fields. For the underlying stochastic process, a very short correlation time of 2 ps has to be assumed, which is tentatively assigned to a flapping motion of the central, nonplanar ring in PSZ. Finally, it has been confirmed by paramagnetic quenching (here Heisenberg exchange) experiments of the magnetic-field effects with TEMPO that all magnetic-field dependencies observed with the present DCAPSZ systems are indeed due to the magnetic-field dependence of spin relaxation.
\end{abstract}

\section{Introduction}

Donor-chromophore-acceptor (DCA) triad systems (vide infra) are a class of supramolecular assemblies that have played an important role in deepening the understanding of photoinitiated electron-transfer processes. These systems are utilized as analogues of natural photosynthesis where nature converts solar energy to chemical energy. Such systems have been studied for many years yielding much information relevant to natural photosynthesis as well as providing insight into possible applications of synthetic photosynthesis mimics in photochemical cells. ${ }^{1-7}$ Our group studies complexes exemplified by the triad in Figure $1 .^{8-11}$ These supramolecular assemblies consist of a central trisbipyridine ruthenium chromophore covalently linked through variable-length polymethylene chains to a "diquat" electron acceptor and a pair of azine-type electron donors. ${ }^{12}$ These triads have proven to be a very fruitful platform for the study of photoinduced electron-transfer processes and charge separation. Specific details about the various triads can be found in the literature, but a brief description of the processes of interest is necessary here before considering the present study. In these triads, a photon of around $450 \mathrm{~nm}$ will directly excite

* To whom correspondence should be addressed. E-mail: elliott@lamar.ColoState.EDU; ulrich.steiner@uni-konstanz.de.

Colorado State University.

$\doteqdot$ Universität Konstanz.

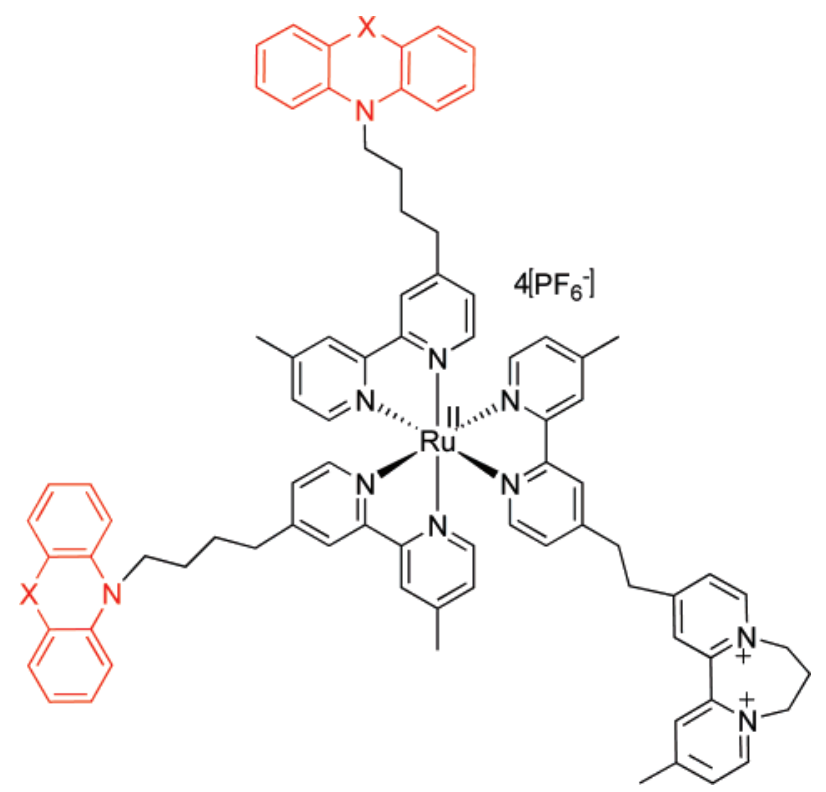

Figure 1. Donor - chromophore-acceptor $(\mathrm{DCA}) \operatorname{triad}(\mathrm{X}=\mathrm{O}, \mathrm{S}$, or Se).

a metal-to-ligand charge-transfer transition (MLCT) in the chromophore. The initial singlet MLCT state ( ${ }^{1}$ MLCT) undergoes rapid intersystem crossing $(<1 \mathrm{ps})$ to the triplet MLCT 


\section{SCHEME 1}

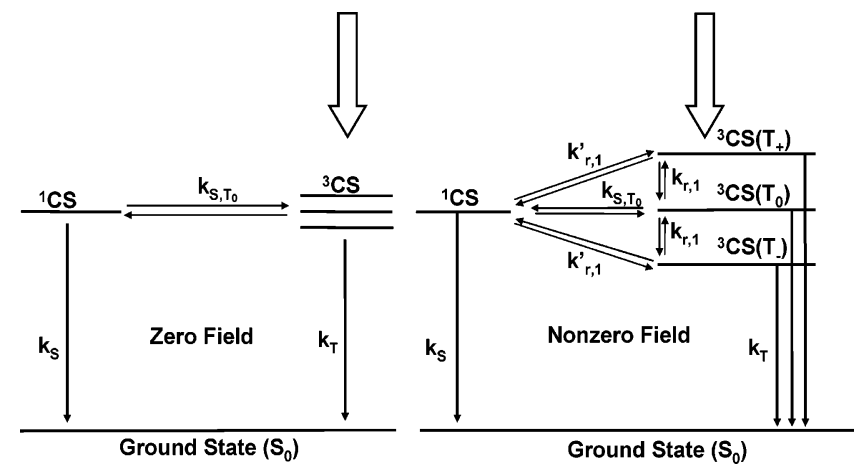

state $\left({ }^{3}\right.$ MLCT). Within a few nanoseconds, a series of electron transfers occur initiating from the ${ }^{3}$ MLCT and yielding a chargeseparated state (CS) wherein the acceptor is reduced; the chromophore is in its original ground state, and one donor is oxidized. Interestingly, in the case where donors are phenothiazine (i.e., $\mathrm{X}=\mathrm{S}$; PTZ), the radical cation pair that constitutes the CS is formed with almost pure triplet spin correlation. ${ }^{8}$ Depending on the structural details of the particular triad complex, this ${ }^{3} \mathrm{CS}$ recombines to the ground state with a lifetime in the range of $100-300 \mathrm{~ns}^{8}$

Spin chemical studies of these triads have proven to be a valuable tool for developing a more sophisticated understanding of the complexities of the CS formation and recombination to the ground state. ${ }^{8}$ Because direct recombination of the ${ }^{3} \mathrm{CS}$ to the singlet ground state is formally a spin-forbidden process, magnetic-field effect (MFE) studies of CS formation and recombination have proven to be of critical importance to the understanding of this spin chemistry. In previous studies (again, involving DCA triads incorporating PTZ donors), the model we have successfully employed to explain the spin chemical behavior of the triad CS is the relaxation mechanism of Hayashi and Nagakura. ${ }^{13}$ Scheme 1 shows the processes operative in ${ }^{3} \mathrm{CS}$ recombination. Because ${ }^{3} \mathrm{CS}$ and ${ }^{1} \mathrm{CS}$ states are essentially degenerate at zero applied magnetic field (vide infra), isotropic hyperfine interaction provides a mechanism for coupling and thus mixing ${ }^{3} \mathrm{CS}$ and ${ }^{1} \mathrm{CS}$ states. In the absence of any applied field, ${ }^{3} \mathrm{CS} /{ }^{1} \mathrm{CS}$ spin equilibration is rapid relative to recombination via the spin-allowed path $k_{\mathrm{s}}$ (which is the only kinetically important route to recombination in zero applied field), resulting in a nearly monoexponential decay of ${ }^{3} \mathrm{CS}$ back to the ground state. ${ }^{8}$ Upon application of a magnetic field, the triplet state experiences Zeeman splitting, as shown in Scheme 1, wherein the $T_{0}$ state energy is unchanged and the energies of the $T_{+}$ and $\mathrm{T}_{-}$states move as indicated. At relatively small fields, the difference in energies of the $\mathrm{T}_{-}$and $\mathrm{T}_{+}$states and those of the ${ }^{1} \mathrm{CS}$ and $\mathrm{T}_{0}$ states becomes significant relative to the hyperfine coupling energy, thus resulting in a slowing of the rate of their conversion to the ${ }^{1} \mathrm{CS}$ state. Stated another way, the coherent isotropic hyperfine coupling is suppressed, resulting in inefficient mixing of the $\mathrm{S}, \mathrm{T}_{+}, \mathrm{T}_{-}$states. Thus, strongly biexponential kinetics develop characterized by a "fast" field-independent component due to $\mathrm{T}_{0}$ recombination and a "slow" fielddependent component corresponding to recombination of the $\mathrm{T}_{-}$and $\mathrm{T}_{+}$states. As the applied field is increased from 0 to 3 $\mathrm{T}$, kinetics of the "slow" component of the CS decay, and thus the mechanism responsible for it, undergoes a transition. At low fields, isotropic hyperfine coupling is still operative in mixing states and allows recombination, albeit progressively less efficiently as the field is increased. At medium fields, a number of other processes start to become potentially important. These are incoherent processes such as anisotropic hyperfine interaction (ahfi), g-tensor anisotropy (gta), spin rotational coupling (src), and electron spin-spin dipolar interaction (esdi). Also, direct spin-orbit coupling (soc) assisted spin-forbidden recombination $k_{\mathrm{T}}$ to the singlet ground state could contribute. ${ }^{8}$

Our previous MFE study yielded much kinetic detail about the formation and recombination of ${ }^{3} \mathrm{CS}$ in one class of triad molecules; however, a number of unanswered questions remain. We were unable to establish which incoherent process or processes dominated recombination at intermediate to high magnetic fields. We established that at moderate fields it was likely that the esdi or ahfi mechanisms were dominant, but we were unable to distinguish between the two. Also, we assumed that a direct spin-forbidden recombination was contributing. This assumption, while reasonable, was not unambiguously verified. Further inquiry is necessary to clearly distinguish among the operative recombination processes across the medium to high field region.

To this end, we have synthesized an expanded series of triad complexes in an attempt to answer some of the questions remaining from our earlier studies. We chose one triad from the previous study, namely, the triad DCA-PTZ shown in Figure 1 where $X=S$, and modified it by changing the chalcogen heteroatom in the donor. A series of three triads results in which only the donor heteroatom differs, where $\mathrm{X}=$ oxygen (DCAPOZ), sulfur (DCA-PTZ), or selenium (DCA-PSZ). These complexes are useful for spin study because they provide a variation of 1 order of magnitude of donor heteroatom soc while remaining very similar in redox properties and essentially isostructural. Variation of soc of this magnitude is expected to dramatically affect processes dominant in recombination at higher fields, specifically direct spin-forbidden recombination, gta, and src. ${ }^{14}$

Finally, several unexpected findings arose in the course of this study that provide a powerful route for strengthening the model. An extreme sensitivity of the MFE of these complexes to paramagnetic spin catalysts (such as molecular oxygen and TEMPO) was observed. Studies have shown that the effect of spin catalysts on the MFE of diradical pairs can provide a means to distinguish the dominant recombination process. ${ }^{15}$ Also, the high field behavior of recombination of the DCA-PSZ complex requires a broadening of the model because none of the mechanisms discussed previously can explain the magnetic-field dependence at fields above $100 \mathrm{mT}$.

\section{Experimental Section}

Materials. 2,2,6,6-Tetramethylpiperidine 1-oxyl (TEMPO). TEMPO (99\%) was purchased from Aldrich and used without further purification.

10-Methylphenoxazine (Me-POZ), 10-Methylphenothiazine (Me-PTZ), and 10-Methylphenoselenazine (Me-PSZ). The synthesis and characterization of Me-POZ and Me-PSZ are reported elsewhere. ${ }^{16,17}$ Me-PTZ was obtained from Aldrich and recrystallized three times from 10:1 toluene-hexanes yielding a colorless crystalline solid.

10-(4-(4'-Methyl-2,2'-bipyridin-4-yl)butyl)-10H-phenoxazine (44-POZ). A procedure from the literature was modified as follows: ${ }^{11}$ in a drybox under $\mathrm{N}_{2}$ atmosphere, phenoxazine $(0.45$ $\mathrm{g}, 2.46 \mathrm{mmol})$ was combined with $\mathrm{NaH}(0.0531 \mathrm{~g}, 1.48 \mathrm{mmol})$, a catalytic amount of NaI, and 4-(4-bromobutyl)-4'-methyl-2,2'bipyidine $(0.5002 \mathrm{~g}, 1.64 \mathrm{mmol})$ in THF (freshly distilled from $\mathrm{Na}$ /benzophenone). ${ }^{11}$ The solution was then refluxed for $12 \mathrm{~h}$, quenched with ethanol, and then dried by rotary evaporation. Upon silica gel chromatography (20:1 methylene chloride- 
acetone), 44-POZ (a colorless oil) was isolated. The compound was characterized with NMR, TLC, and electrospray mass spectroscopy ( $\mathrm{M}+\mathrm{H} 408.3)$.

10-(4-(4'-Methyl-2,2'-bipyridin-4-yl)butyl)-10H-phenoselenazine (44-PSZ). The compound was prepared, isolated, and characterized by a method analogous to that of 44-POZ given above: phenoselenazine (0.2446 g, $9.93 \mathrm{mmol})$; $\mathrm{NaH}(0.0215$ g, 8.96 mmol); 4-(4-bromobutyl)-4'-methyl-2,2'-bipyidine (0.202 $\mathrm{g}, 6.62 \mathrm{mmol})$; mass spectroscopy $(\mathrm{M}+2 \mathrm{H} 472.2) .{ }^{11}$

$(423-D Q)\left(P_{6}\right)_{2}$. A procedure from the literature was modified as follows: ${ }^{11} 1,2$ bis[4-(4-methyl-2,2'-bipyridyl)] ethane $(0.70 \mathrm{~g}, 1.91 \mathrm{mmol})$ was dissolved in $n$-heptane with a large excess of diiodopropane (purified over alumina), and the solution was refluxed for 6 days. The crude product, (423-DQ)(I) 2 , was isolated as a red solid, dissolved in water, and precipitated as the $\mathrm{PF}_{6}{ }^{-}$salt. Pure (423-DQ) $\left(\mathrm{PF}_{6}\right)_{2}$ was isolated as a purple oil from this solid using Soxhlet extraction with methanol. ${ }^{18,19}$ The product was characterized using TLC (eluent $40 \% \mathrm{H}_{2} \mathrm{O}-10 \%$ $\mathrm{KNO}_{3}$ (aq satd)-50\% acetonitrile (4:1:5(vol)) and electrospray mass spectroscopy ( $\mathrm{M}+\mathrm{H}$ 699.4).

$\mathrm{Ru}(44-\mathrm{POZ}) \mathrm{Cl}_{2}$. A procedure from the literature was modified as follows: ${ }^{11}$ in a drybox under $\mathrm{N}_{2}$ atmosphere, $\mathrm{Ru}(\mathrm{DMSO})_{4}{ }^{-}$ $\mathrm{Cl}_{2}$ (0.1947 g, $\left.0.402 \mathrm{mmol}\right)$ and $\mathrm{LiCl}(0.170 \mathrm{~g}, 4.01 \mathrm{mmol})$ were dissolved in DMF (dried over molecular sieves) and refluxed until an orange color appeared (30 min); 44-POZ (0.327 g, 0.803 mmol) dissolved in minimum DMF was added over several minutes, and the solution was refluxed for $90 \mathrm{~min}$. After removing from the drybox, upon doubling the volume with $\mathrm{H}_{2} \mathrm{O}$, a purple solid precipitated, which, upon silica gel chromatography (10:1 methylene chloride-methanol saturated with ammonia), yielded $\mathrm{Ru}(44-\mathrm{POZ}) \mathrm{Cl}_{2}$ as a purple oil. Light was rigorously excluded throughout the above procedure. ${ }^{11}$

$\mathrm{Ru}(44-\mathrm{POZ})_{2}(423-\mathrm{DQ})\left(\mathrm{PF}_{6}\right)_{4}$. A procedure from the literature was modified as follows: ${ }^{11}$ in a drybox under $\mathrm{N}_{2}$ atmosphere $\mathrm{Ru}(44-\mathrm{POZ})_{2} \mathrm{Cl}_{2}(0.061 \mathrm{~g}, 0.062 \mathrm{mmol})$ was heated to $120^{\circ} \mathrm{C}$ in ethylene glycol for $30 \mathrm{~min}$; (423-DQ) $\left(\mathrm{PF}_{6}\right)_{2}(0.0561 \mathrm{~g}, 0.080$ $\mathrm{mmol}$ ) dissolved in acetone, was added, and the solution was heated for $30 \mathrm{~min}$ at $120^{\circ} \mathrm{C}$. The mixture was removed from the drybox, diluted with $\mathrm{H}_{2} \mathrm{O}$, and separated as $\mathrm{PF}_{6}{ }^{-}$salt using centrifugation; silica gel chromatography (eluent $40 \% \mathrm{H}_{2} \mathrm{O}-10 \%$ $\mathrm{KNO}_{3}$ (aq satd)-50\% acetonitrile (4:1:5(vol)) yielded the product as a dark solid. Light was rigorously excluded throughout the above procedure. ${ }^{11}$ A combination of TLC, electrospray mass spectroscopy ( $M+1$ 1759), and electrochemistry was used for determination of sample integrity.

$\mathrm{Ru}(44-\mathrm{PSZ})_{2} \mathrm{Cl}_{2}$. The compound was prepared, isolated, and characterized by a method analogous to that of $\mathrm{Ru}(44-\mathrm{POZ})$ $\mathrm{Cl}_{2}$ given above: $\mathrm{Ru}(\mathrm{DMSO})_{4} \mathrm{Cl}_{2}(0.0824 \mathrm{~g}, 0.170 \mathrm{mmol}) ; \mathrm{LiCl}$ (0.150 g, $3.54 \mathrm{mmol})$; 44-PSZ (0.1683 g, $0.3579 \mathrm{mmol})$.

$R u(44-P S Z)_{2}(423-D Q)\left(P F_{6}\right)_{4}$. The compound was prepared, isolated, and characterized by a method analogous to that of $\mathrm{Ru}(44-\mathrm{POZ})_{2}(423-\mathrm{DQ})\left(\mathrm{PF}_{6}\right)_{4}$ given above: $\mathrm{Ru}(44-\mathrm{PSZ})_{2} \mathrm{Cl}_{2}$ (0.0796 g, $0.0715 \mathrm{mmol})$; (423-DQ) $\left(\mathrm{PF}_{6}\right)_{2}(0.06497 \mathrm{~g}, 0.093$ $\mathrm{mmol})$; electrospray mass spectroscopy $(\mathrm{M}+1$ 1885).

$R u(44-P T Z)_{2}(423-D Q)\left(P F_{6}\right)_{4}$. The preparation of this compound was reported previously. ${ }^{11}$

Preparation of Samples for Kinetic Studies. Samples for kinetic studies were initially prepared in cells as described previously using multiple freeze-pump-thaw cycles to remove dissolved oxygen. ${ }^{9}$ Samples consisted of a $2.5 \times 10^{-5} \mathrm{M}$ solution of the complex in 1,2 dichloroethane (Acros). It was found that a further deoxygenation step was necessary to obtain truly reproducible kinetics with moderate applied magnetic fields (>300 mT). Once freeze-pump-thaw degassed, the samples were taken into a nitrogen-filled drybox (below 1 ppm oxygen) and allowed to equilibrate with the box atmosphere (30 min).

Measurements. Cyclic Voltammetry (CV). A conventional three-electrode electrochemical cell with a BAS 100B electrochemical analyzer was used for all CV measurements. $0.1 \mathrm{M}$ tetra- $n$-butylammonium hexafluorophosphate $\left(\mathrm{TBAPF}_{6}\right)$ in acetonitrile was used as the electrolyte. A glassy carbon working electrode was used along with a platinum wire auxiliary electrode and a saturated sodium calomel (SSCE) reference electrode. A scan rate of $200 \mathrm{mV} / \mathrm{s}$ was used for all measurements. All solutions were purged with argon prior to electrochemical experiments.

Spectroelectrochemistry. The optically transparent thin layer electrochemical cell (OTTLE) was adapted from the literature. ${ }^{20}$ The electrochemical cell consisted of an optically transparent gold minigrid working electrode $\left(4.33 \times 10^{-2} \mathrm{~cm}\right.$ path length), a platinum wire auxiliary electrode, and a silver wire quasireference electrode. The electrolyte solution consisted of a $1 \mathrm{M}$ tetraethylammonium perchlorate $\left(\mathrm{TEAClO}_{4}\right)$ solution in acetonitrile. Samples with concentrations of $10^{-3} \mathrm{M}$ of the free donor (Me-POZ, Me-PTZ, or Me-PSZ) in electrolyte solution were injected into the optically transparent portion of the cell, and a potential $100 \mathrm{mV}$ positive of the first oxidation wave for the donor was applied. The cell was placed perpendicular to the optical train of a Hewlett-Packard 8452A UV-visible spectrometer, and electronic spectra were taken at $1 \mathrm{~s}$ intervals until the current passing through the cell dropped to a minimal level (usually around $60 \mathrm{~s}$ ). Extinction coefficient spectra were calculated for the oxidized donors from the resulting data.

Nanosecond Laser Flash Photolysis. A typical magnetic-fielddependent transient spectrum was obtained as follows: The frequency-tripled beam of a Quanta-Ray Nd:YAG laser pumped a Spectra Physics PDL-3 dye laser. Coumarin 450 laser dye was used with methanol, which was operated at $450 \mathrm{~nm}$. Dye laser power output was typically $85 \mathrm{~mW}$ at $30 \mathrm{~Hz}$ with a 5-7 ns pulse width. The probe beam was provided by a home-built pulser powering a Xenon arc lamp (1 ms pulses). This probe beam was passed through the sample cell and then focused onto the slit of a Jarrell Ash model 82-310 monochromator. A Hamamatsu R2496 photomultiplier tube measured the intensity of the probe beam, and a Tektronix oscilloscope triggered by a Thorlabs DET210 photodiode was used to record and display the data. The experiment was run at $30 \mathrm{~Hz}$, and transient signals were averaged over 500 pulses. Magnetic-field effect measurements were collected by the placement of the sample between the poles of a Walker Scientific Inc. HV4H electromagnet (Hewlett-Packard 6574A power supply) and routing the optical train through the sample. The magnetic field was applied perpendicular to the optical path. Magnetic induction was measured with a Hall probe (F. W. Bell, model 5080).

\section{Results}

Prior to photoexcitation, only the chromophore moiety of DCA triads exhibits any significant visible absorbance above ca. $375 \mathrm{~nm}$. In contrast, each oxidized donor and the reduced acceptor have various strong absorption bands across the visible spectral region. Figure 2 shows spectra of oxidized donors Me$\mathrm{POZ}^{+\bullet}, \mathrm{Me}-\mathrm{PTZ}^{+\bullet}$, and Me-PSZ ${ }^{+\bullet}$, obtained using an optically transparent thin layer electrode spectroelectrochemical cell. Each donor exhibits a strong increase in absorbance around $520 \mathrm{~nm}$ upon oxidation. Upon reduction, the acceptor moiety also develops a number of absorption bands, the strongest appearing at $388 \mathrm{~nm} .{ }^{8,21}$ In our earlier study with DCA-PTZ, we showed that the transient absorption spectrum of the CS could be 


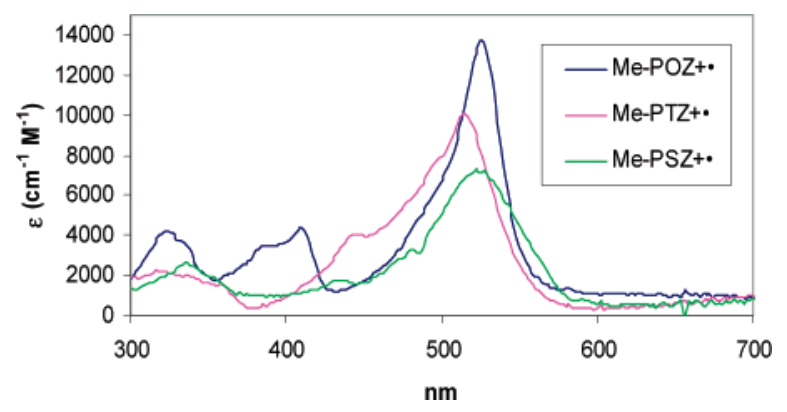

Figure 2. Extinction coefficient spectra for the radical cation of each donor. At the maximum absorbance near $520 \mathrm{~nm}$, the respective values of $\epsilon$ obtained are $14360 \pm 1800$ (Me-POZ), $10008 \pm 315$ (Me-PTZ), and $7531 \pm 582$ (Me-PSZ).
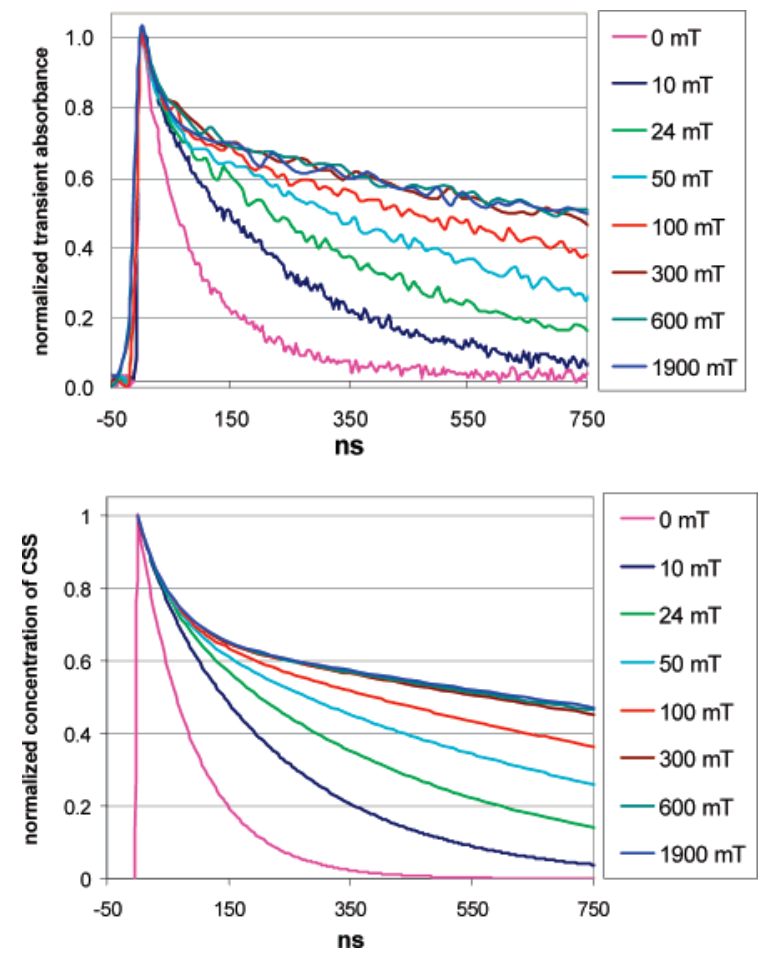

Figure 3. Transient decays for CS of DCA-POZ. Upper panel: experimental curves. Lower panel: simulation using the relaxation scheme with parameters $k_{\mathrm{S}}=3.5 \times 10^{7} \mathrm{~s}^{-1}, k_{\mathrm{T}}=0, k_{\mathrm{r}, 10}=6.4 \times 10^{6}$ $\mathrm{s}^{-1}, k_{\mathrm{r}, 25}=2.8 \times 10^{6} \mathrm{~s}^{-1}, k_{\mathrm{r}, 50}=1.5 \times 10^{6} \mathrm{~s}^{-1}, k_{\mathrm{r}, 100}=9.3 \times 10^{5} \mathrm{~s}^{-1}$, $k_{\mathrm{r}, 300}=5.7 \times 10^{5} \mathrm{~s}^{-1}, k_{\mathrm{r}, 600}=5.4 \times 10^{5} \mathrm{~s}^{-1}$, and $k_{\mathrm{r}, 1900}=5.1 \times 10^{5}$ $\mathrm{s}^{-1}$. (For zero field, a monoexponential with $k=1.1 \times 10^{7} \mathrm{~s}^{-1}$ is shown).

faithfully reproduced by summing equally weighted individual spectra of Me-PTZ ${ }^{+\bullet}$ and $\mathrm{DQ}^{+\bullet} .8,21$ Therefore, transient absorption measurements at either 388 or $520 \mathrm{~nm}$ should show identical CS decay rates as is indeed observed.

Decay profiles for the CS of DCA-POZ and DCA-PSZ are shown in Figures $3 \mathrm{a}$ and $4 \mathrm{a}$, respectively. As expected from previous nanosecond and picosecond studies, all decays show "instantaneous" formation of the CS upon laser excitation (i.e., well within the $6 \mathrm{~ns}$ fwhm laser pulse). ${ }^{8}$ The zero field decay for each of the complexes deviates slightly from monoexponential behavior due to the presence of several geometric isomers of each triad. ${ }^{22}$

With increasing magnetic field, all three complexes undergo qualitatively similar changes in CS decay. Between 0 and ca. $500 \mathrm{mT}$, decays become distinctly biexponential. In this field regime, the rate of the major component progressively decreases with increased field and the rate of the minor component remains unchanged. DCA-POZ and DCA-PTZ triads show almost
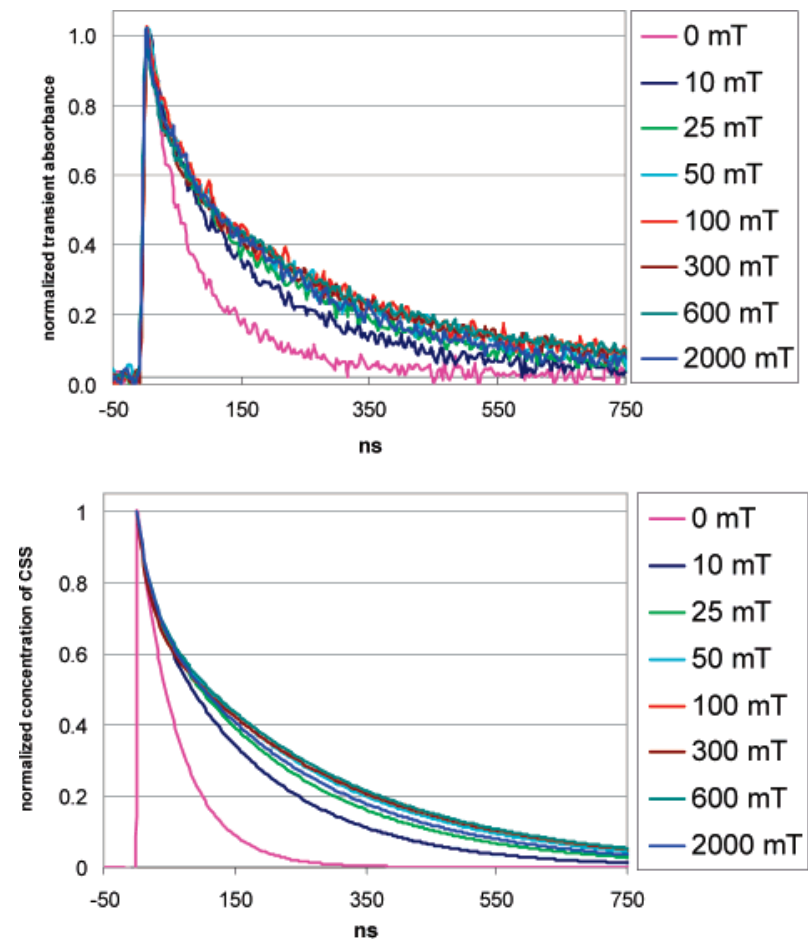

Figure 4. Transient decays for CS of DCA-PSZ. Upper panel: experimental curves. Lower panel: simulation using the relaxation scheme with parameters $k_{\mathrm{s}}=7.6 \times 10^{7} \mathrm{~s}^{-1}, k_{\mathrm{T}}=1.0 \times 10^{6} \mathrm{~s}^{-1}, k_{\mathrm{r}, 0}=$ $5.00 \times 10^{7} \mathrm{~s}^{-1}, k_{\mathrm{r}, 10}=5.39 \times 10^{6} \mathrm{~s}^{-1}, k_{\mathrm{r}, 25}=3.83 \times 10^{6} \mathrm{~s}^{-1}, k_{\mathrm{r}, 50}=$ $3.00 \times 10^{6} \mathrm{~s}^{-1}, k_{\mathrm{r}, 100}=2.72 \times 10^{6} \mathrm{~s}^{-1}, k_{\mathrm{r}, 300}=2.71 \times 10^{6} \mathrm{~s}^{-1}, k_{\mathrm{r}, 600}=$ $2.71 \times 10^{6} \mathrm{~s}^{-1}$, and $k_{\mathrm{r}, 2000}=3.42 \times 10^{6} \mathrm{~s}^{-1}$.

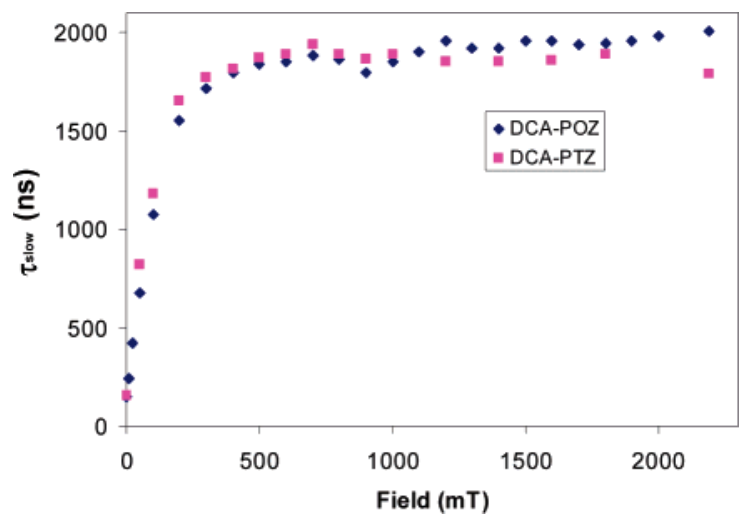

Figure 5. $\tau_{\text {slow }}$ vs $B$ for DCA-PTZ and DCA-POZ.

identical MFEs with increasing field up to a saturation limit of about $500 \mathrm{mT}$ (Figure 5). The DCA-PSZ, however, shows qualitatively similar behavior except that the rate constant of the slow component is an order of magnitude larger (Figure 6). Also, the time constant of the slow component passes through a maximum between 100 and $400 \mathrm{mT}$ and shows a definite decrease with increasing field up to at least $3000 \mathrm{mT}^{23}$

In our earlier study of DCA-PTZ and other PTZ containing triads, we were able to show that the amount of CS initially formed upon photoexcitation has a subtle field dependence above $500 \mathrm{mT}$ (i.e., $<10 \%$ decrease between 500 and 3000 mT) ${ }^{8}$ We presume that similar changes may exist with DCAPOZ and DCA-PSZ; however, because of issues in the present studies with long-term power stability of the dye laser, we were unable to experimentally verify this assumption.

Finally, in the course of these studies, we observed that the MFEs for all of these compounds are extremely sensitive to trace $\mathrm{O}_{2}$, so much so that we were unable to obtain quantitatively 


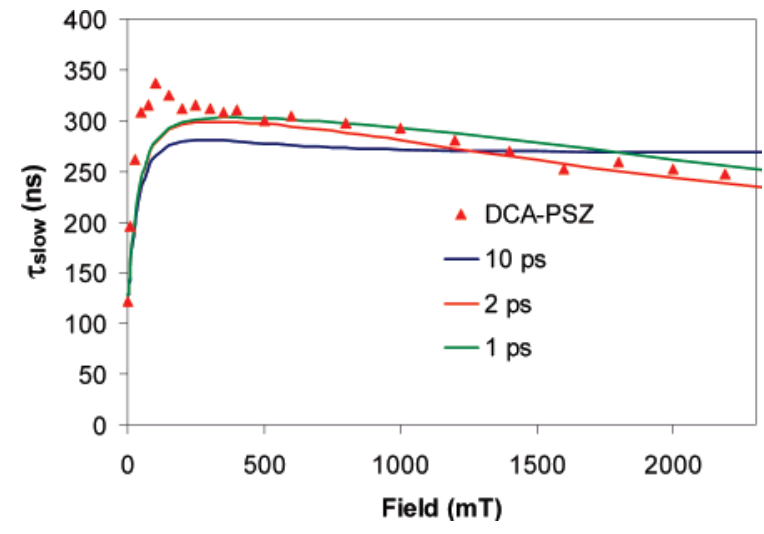

Figure 6. $\tau_{\text {slow }}$ vs $B$ for DCA-PSZ. The solid lines correspond to theoretical predictions according to the gta mechanism and adopting values of 10,2 , and $1 \mathrm{ps}$ for the orientational correlation time as indicated. For the calculation of the theoretical curves, cf. discussion.

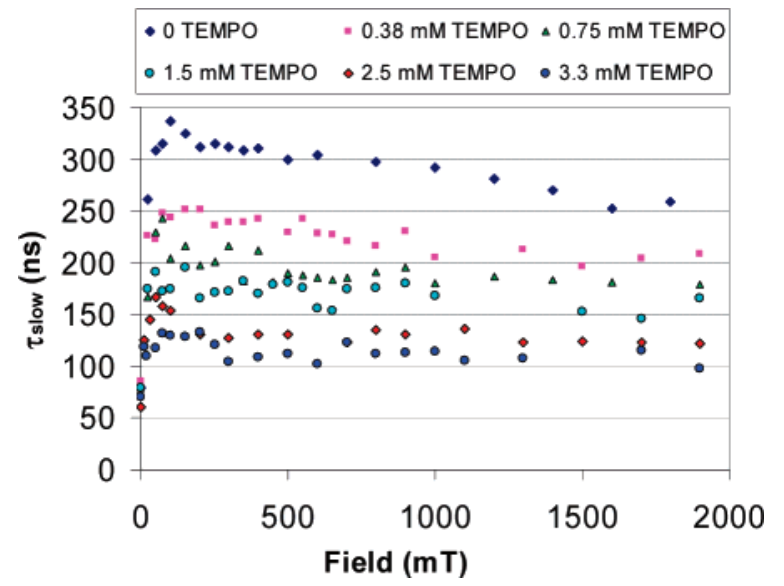

Figure 7. Tempo effect on $\tau_{\text {slow }}$ for DCA-PSZ.

reproducible CS decay rates when we attempted to remove dissolved $\mathrm{O}_{2}$ by multiple freeze-pump-thaw cycles on a Schlenk line. Only when sample cells were loaded in a $\mathrm{N}_{2}$ inert atmosphere box having an $\mathrm{O}_{2}$ concentration of $<1 \mathrm{ppm}$ were we able to obtain consistently reproducible rates. ${ }^{24}$ The observation of this extreme $\mathrm{O}_{2}$ sensitivity led us to study the effects of a spin catalyst, specifically TEMPO, on the MFE for the DCAPSZ triad. Figure 7 shows plots of $\tau_{\text {slow }}$ as a function of field at a series of TEMPO concentrations. As this data shows, at about $3 \mathrm{mM}$ TEMPO the MFE on this triad is essentially obliterated.

\section{Discussion}

The kinetic analysis of the observed magnetic-field dependence of the recombination kinetics of the CS state must be based on a detailed spin substate-dependent reaction scheme as depicted in Scheme 1. As has been shown previously, ${ }^{8}$ in magnetic fields below $1 \mathrm{~T}$ it can be assumed that the CS state originates with almost pure triplet spin. The CS state represents a radical pair (RP) with an average separation of more than 10 $\AA$ so that exchange interaction is negligible for most conformations of the chains linking the radical moieties to the bipyridine ligands. Therefore, in zero magnetic field all of the spin substates are degenerate and the three triplet substates are kinetically equivalent. ${ }^{25}$ For the radical species we are dealing with here, the most effective mechanism for triplet-singlet transitions in the CS state is due to isotropic hyperfine interaction causing a coherent triplet-singlet mixing process at a rate that can be
TABLE 1: EPR Parameters ${ }^{a}$ of Me-PXZ Cation Radicals ${ }^{17}$

\begin{tabular}{llll}
\hline & Me-POZ & Me-PTZ & Me-PSZ \\
\hline$a(1 \mathrm{~N})$ & 9.18 & 7.56 & 6.82 \\
$a(3 \mathrm{H})$ & 9.07 & 7.22 & 7.30 \\
$a(2 \mathrm{H})$ & 2.99 & 2.17 & 2.13 \\
$a(2 \mathrm{H})$ & 1.49 & 0.96 & 0.92 \\
$a(2 \mathrm{H})$ & 0.56 & 0.75 & 0.77 \\
$a(2 \mathrm{H})$ & 0.57 & 0.31 & 0.43 \\
$a\left({ }^{77} \mathrm{Se}\right)^{b}$ & & & 23.5 \\
$g$ & 2.0040 & 2.0052 & 2.0153 \\
line width & 0.30 & 0.30 & 0.55
\end{tabular}

${ }^{a}$ Hyperfine couplings and line widths in gauss. ${ }^{b}$ Natural abundance of $7.6 \%$.

estimated by the semiclassical effective hyperfine field $B_{1 / 2}$ defined as ${ }^{26,27}$

$$
B_{1 / 2}=\left(3 \sum_{i} a_{i}^{2} I_{i}\left(I_{i}+1\right)\right)^{1 / 2}
$$

where $a_{i}$ and $I_{i}$ are the isotropic hyperfine coupling constant and nuclear spin quantum number of a nucleus and the index $i$ runs over all magnetic nuclei of the RP. For the CS state with PTZ, a value of $2.82 \mathrm{mT}$ is obtained. Because the hyperfine coupling constants for the radicals of POZ and PSZ are very similar to that of PTZ (cf. Table 1), the $B_{1 / 2}$ value is essentially the same for the three systems. It corresponds to a time constant of about 2 ns, that is, a very short time scale in relation to the time scale of the observed kinetics. So, in zero field, established spin equilibrium between all spin sublevels can be assumed. As the field is increased above the value of $B_{1 / 2}$, the Zeeman splitting of $\mathrm{T}_{+}$and $\mathrm{T}_{-}$exceeds the typical hyperfine coupling and the coherent spin transitions between $T_{ \pm}, T_{0}$, and $S$ are quenched, whereas the coherent transitions between $\mathrm{T}_{0}$ and $\mathrm{S}$ are not affected. On a longer time scale, transitions between $\mathrm{T}_{ \pm}$and $\mathrm{T}_{0}, \mathrm{~S}$ are brought about in an incoherent fashion through the individual longitudinal $\left(\mathrm{T}_{1}\right)$ spin relaxation of the radical spins or through their combined $\mathrm{T}_{ \pm} \rightarrow \mathrm{T}_{0}$ relaxation caused by dipolar electron spin-spin interaction. The rates of these processes are magnetic-field-dependent at least to some degree so that a magnetic-field effect on the overall recombination kinetics of the CS state results.

It is appropriate to describe the kinetics in terms of coupled rate equations for relaxation and reaction as originally suggested by Hayashi and Nagakura ${ }^{13}$ and also employed in our previous investigation. ${ }^{8}$ The rate constants assigned in Scheme 1 are the following: $k_{\mathrm{S}}$ and $k_{\mathrm{T}}$ denote the rate constants of direct recombination of the singlet and triplet substates of $\mathrm{CS}$, respectively. For the singlet state ${ }^{1} \mathrm{CS}$, this process is spinallowed and therefore much faster than that for the triplet substates ${ }^{3} \mathrm{CS}$. In the latter case, spin conversion must go along with the backward transfer of the electron, which is only possible under special conditions with enhanced spin-orbit coupling. The rate constants $k_{\mathrm{r}, 1}$ and $k_{\mathrm{r}, 1}{ }^{\prime}$ characterize the relaxation transitions $\mathrm{T}_{ \pm} \rightarrow \mathrm{T}_{0}$ and $\mathrm{T}_{ \pm} \rightarrow \mathrm{S}$, respectively. The effective sum of all processes contributing to the establishment of the $\mathrm{T}_{0}$ $\rightarrow \mathrm{S}$ equilibrium is denoted by $k_{\mathrm{S}, \mathrm{T}_{0}}$. It is assumed that $k_{\mathrm{S}, \mathrm{T}_{0}}>$ $>k_{\mathrm{S}}, k_{\mathrm{T}}$ so that this equilibrium is maintained during all stages of the recombination of CS. For this reason, the rate constants $k_{\mathrm{r}, 1}$ and $k_{\mathrm{r}, 1}{ }^{\prime}$ can be combined and only their sum $k_{\mathrm{r}} \equiv k_{\mathrm{r}, 1}+$ $k_{\mathrm{r}, 1^{\prime}}$ enters the kinetic result.

Assuming initial population of the ${ }^{3} \mathrm{CS}$ states only, the decay kinetics of CS is described by ${ }^{8}$

$$
[C S]=[C S]_{0}\left(c_{a} \mathrm{e}^{-k_{a} t}+c_{b} \mathrm{e}^{-k_{b} t}\right)
$$




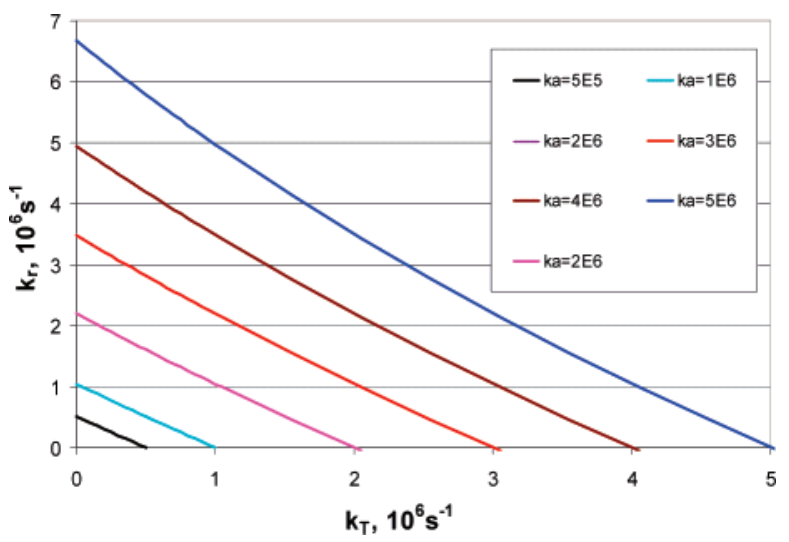

Figure 8. Representation of $k_{\mathrm{r}}$ and $k_{\mathrm{T}}$ pairs that are compatible with the same value of $k_{a}$ and yield the exact same quality of the kinetic fit $\left(k_{\mathrm{S}}=5 \times 10^{7} \mathrm{~s}^{-1}\right)$.

The corresponding equation holds for the transient absorbance $A$ if $[C S]$ is replaced by $A$

$$
\begin{gathered}
A=A_{0}\left(c_{a} \mathrm{e}^{-k_{a} t}+c_{b} \mathrm{e}^{-k_{b} t}\right) \\
\text { with } c_{a}+c_{b}=1 \\
c_{a}=\frac{1}{2}+\frac{3 k_{\mathrm{r}}+\left(k_{\mathrm{S}}-k_{\mathrm{T}}\right) / 4}{6 \sqrt{k_{\mathrm{r}}^{2}+\left(k_{\mathrm{S}}-k_{\mathrm{T}}\right)^{2} / 16}} \\
k_{a}=k_{\mathrm{r}}+\frac{1}{4} k_{\mathrm{S}}+\frac{3}{4} k_{\mathrm{T}}-\sqrt{k_{\mathrm{r}}^{2}+\left(k_{\mathrm{S}}-k_{\mathrm{T}}\right)^{2} / 16} \\
k_{b}=k_{\mathrm{r}}+\frac{1}{4} k_{\mathrm{S}}+\frac{3}{4} k_{\mathrm{T}}+\sqrt{k_{\mathrm{r}}^{2}+\left(k_{\mathrm{S}}-k_{\mathrm{T}}\right)^{2} / 16}
\end{gathered}
$$

Equation $2 \mathrm{~b}$ represents a biexponential decay function with four independent parameters, viz., $A_{o}, c_{a}, k_{a}, k_{b}$. Variations of $A_{o}$ are due to variations in the laser intensity and can be eliminated by normalization of the signal curves. The remaining three degrees of freedom can be represented by the rate constants $k_{\mathrm{S}}, k_{\mathrm{T}}$, and $k_{\mathrm{r}}$. Of these, only $k_{\mathrm{r}}$ is expected to be magneticfield-dependent. Thus, in fitting a set of normalized kinetic traces for a certain sample for varying magnetic fields, $k_{\mathrm{S}}$ and $k_{\mathrm{T}}$ should be kept fixed as global parameters and only $k_{\mathrm{r}}$ is free for fitting the complete decay curve. This is a strong criterion for the validity of the kinetic model. As is shown in Figures 3 and 4, the procedure describes the complete behavior of the observed decay curves very well.

According to Scheme 1, there are two channels, the " $k_{\mathrm{r}}$ channel" and the " $k_{\mathrm{T}}$ channel", through which ${ }^{3} \mathrm{CS}$ can recombine. Although $k_{\mathrm{r}}$ and $k_{\mathrm{T}}$ do not enter eqs $4-6$ in an equivalent manner (for the phenomenological parameters of the biexponential), their influence becomes essentially indistinguishable if only kinetic reasoning is invoked. If $k_{\mathrm{S}} \gg k_{\mathrm{r}}, k_{\mathrm{T}}$, then $k_{a}$ $\rightarrow\left(k_{\mathrm{r}}+k_{\mathrm{T}}\right)$. For $k_{\mathrm{S}} \approx 5 \times 10^{7} \mathrm{~s}^{-1}$, as is the typical order of magnitude for the systems under consideration, this is not exact and one has to resort to the general equation

$$
k_{\mathrm{r}}=\frac{\left(k_{\mathrm{S}}-k_{\mathrm{T}}\right)^{2} / 16-\left(\frac{1}{4}\left(k_{\mathrm{S}}+3 k_{\mathrm{T}}\right)-k_{a}\right)^{2}}{2\left(\frac{1}{4}\left(k_{\mathrm{S}}+3 k_{\mathrm{T}}\right)-k_{a}\right)}
$$

to obtain kinetically equivalent pairs of $k_{\mathrm{r}}$ and $k_{\mathrm{T}}$ for a certain value of $k_{a}{ }^{28}$ The relation between equivalent pairs of $k_{\mathrm{r}}$ and $k_{\mathrm{T}}$ for fixed values of $k_{a}$ is shown in Figure 8, which will be

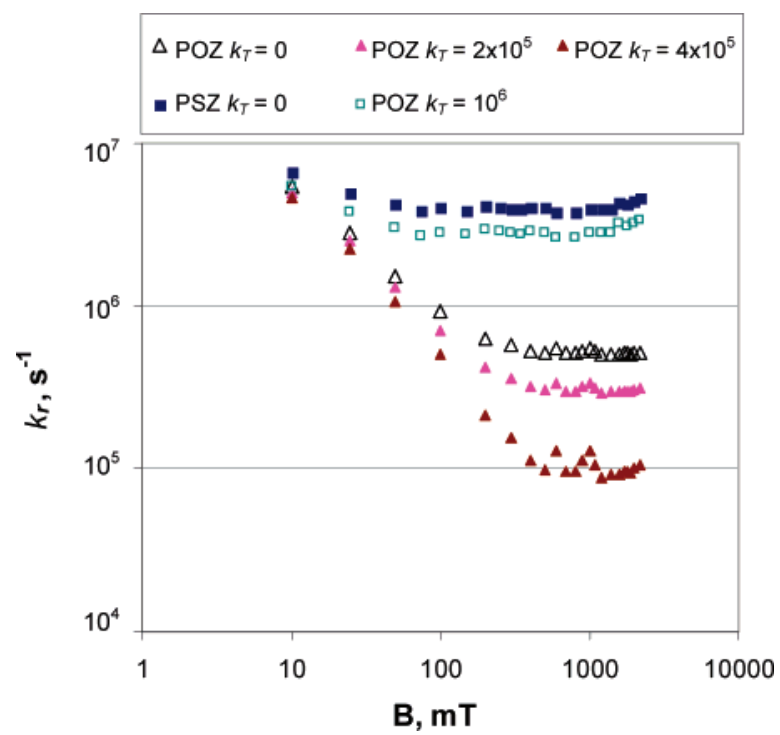

Figure 9. Magnetic-field dependence of $k_{\mathrm{r}}$ as obtained from fitting the signal decays for DCA-POZ and DCA-PSZ (cf. Figures 3 and 4) for different values of $k_{\mathrm{T}}$.

used when criteria limiting the sensible range of values of either $k_{\mathrm{r}}$ or $k_{\mathrm{T}}$ are at hand.

The rate constant $k_{\mathrm{r}}$ may be generally decomposed into a magnetic-field-dependent contribution $k_{\mathrm{r}, B}$ and a magnetic-field independent contribution $k_{\mathrm{r}, \mathrm{c}}$, viz.

$$
k_{\mathrm{r}}(B)=k_{\mathrm{r}, B}(B)+k_{\mathrm{r}, \mathrm{c}}
$$

Through its magnetic-field dependence, $k_{\mathrm{r}, B}(B)$ can be separated from $k_{\mathrm{r}, \mathrm{c}}$. So the problem of separating $k_{\mathrm{r}}$ and $k_{\mathrm{T}}$ is reduced to a separation of $k_{\mathrm{r}, \mathrm{c}}$ and $k_{\mathrm{T}}$, that is, to the situation prevailing at high fields.

The magnetic-field dependence of the $k_{\mathrm{r}}$ values for DCAPOZ and DCA-PSZ obtained from fitting the decay curves by assuming specific values for $k_{\mathrm{T}}$ are shown in Figure 9. No data points for DCA-PTZ are shown here because they are actually very close to the results for DCA-POZ. As becomes clear from Figure 9, the results for $k_{\mathrm{r}}$ depend to a significant extent on the assumed value of $k_{\mathrm{T}}$. The quality of the fits is independent of the value assumed for $k_{\mathrm{T}}$ and thus does not provide a criterion for narrowing the bounds of this parameter. There are, however, other criteria at hand.

As the value of $k_{\mathrm{T}}$ is increased, the value of $k_{\mathrm{r}}$ decreases. Obviously, for DCA-POZ the relative change of $k_{\mathrm{r}}$ with $k_{\mathrm{T}}$ is small for large values of $k_{\mathrm{r}}$ (low fields) and large for small values of $k_{\mathrm{r}}$ (high fields). As can be seen in Figure 9, for $k_{\mathrm{T}}=10^{6} \mathrm{~s}^{-1}$ the low field value of $k_{\mathrm{r}}$ for DCA-PSZ equals those for DCAPOZ (cf. the data points at $10 \mathrm{mT}$, which converge for the $k_{\mathrm{T}}$ values adopted for DCA-POZ). For higher values of $k_{\mathrm{T}}$ (not shown) the $k_{\mathrm{r}}$ value for DCA-PSZ would even drop below the $k_{\mathrm{r}}$ values of DCA-POZ. Such a result is not sensible, however, because spin relaxation in PSZ must be faster than that for POZ, as is qualitatively borne out by the EPR linewidths of the cation radicals (cf. Table 1) and as to be expected from the increased spin-orbit coupling of selenium with respect to oxygen. Thus, $k_{\mathrm{T}}=10^{6} \mathrm{~s}^{-1}$ seems to represent an upper bound for DCA-PSZ. However, this limit can still be lowered. If we assume that all relaxation mechanisms operating in the DQ-POZ radical pair are also effective in the DQ-PSZ pair and we add to the 
TABLE 2: Contributions to the Relaxation Rate Constant $\boldsymbol{k}_{\mathrm{r}}$

\begin{tabular}{lc}
\hline \multicolumn{1}{c}{ mechanism } & rate constant \\
\hline anisotropic hyperfine interaction (ahfi) & $k_{\mathrm{r}, \mathrm{a}}$ \\
$g$ tensor anisotropy (gta) & $k_{\mathrm{r}, \mathrm{g}}$ \\
combined action of ahfi and gta & $k_{\mathrm{r}, \mathrm{ag}}$ \\
spin-rotational interaction & $k_{\mathrm{r}, \mathrm{sri}}$ \\
electron spin dipolar interaction & $k_{\mathrm{r}, \mathrm{esdi}}$
\end{tabular}

experimental $k_{\mathrm{r}}$ values of the former the field-independent contribution $\left(3.8 \times 10^{6} \mathrm{~s}^{-1}\right)$ of the latter, then the resulting values exceed the $k_{\mathrm{r}}$ values for DQ-PSZ obtained under the assumption $k_{\mathrm{T}}=10^{6} \mathrm{~s}^{-1}$. Actually, in the case of DQ-PSZ the possible contribution of $k_{\mathrm{T}}$ must be within the experimental accuracy limit.

The relative strength of spin-orbit coupling in oxygen and selenium provides an argument for an upper bound of $k_{\mathrm{T}}$ for POZ. The process to which $k_{\mathrm{T}}$ refers, that is, direct recombination of the ${ }^{3} \mathrm{CS}$ state to the singlet ground state, requires a spin flip during electron transfer. As may be concluded from the way they can be influenced by heavy atom substituents, such processes have been shown to be controlled by spin-orbit coupling. ${ }^{29}$ A proportionality of the rate constant to the square of the atomic spin-orbit coupling constant has been observed.

Considering that the atomic spin-orbit coupling constants of selenium and oxygen are in the ratio of 1990:158 $=12.6$, the $k_{\mathrm{T}}$ value for PSZ is expected to be about 150 larger than for POZ. Thus, the upper bound of $k_{\mathrm{T}}$ for POZ would be established at about $10^{4} \mathrm{~s}^{-1}$. Such a small value of $k_{\mathrm{T}}$ is indistinguishable from $k_{\mathrm{T}}=0$ in the $k_{\mathrm{r}}$ curves obtained from the kinetic fits and one can realistically assume that $k_{\mathrm{T}}$ is negligible for POZ and hence also for PTZ because the experimental results for it are almost indistinguishable from those for POZ.

The fact that soc in the donor radical is in fact governed by the heteroatom center is also supported by the observed $g$ values of the Me-PXZ cation radicals. It has been established ${ }^{30}$ that the deviation of the $g$ factor from the value $g_{\mathrm{e}}$ of the free electron $\left(g-g_{\mathrm{e}}\right)$ is proportional to the spin-orbit coupling constant $\zeta$ if spin-orbit coupling at one particular atomic center is responsible for this deviation. The values of $\left(g-g_{\mathrm{e}}\right)$ for Me-POZ, Me-PTZ, and Me-PSZ were found in the ratio 0.59:1:4.5 (cf. Table 1), which is in reasonable agreement with the atomic spin-orbit coupling constants of $\mathrm{O}, \mathrm{S}$, and $\mathrm{Se}(158: 396: 1990=0.4: 1$ : 5.0). ${ }^{31,32}$

Theoretical Estimation of Various Contributions to $\boldsymbol{k}_{\mathrm{r}}$. The contributions to the relaxation rate constant $k_{\mathrm{r}}$ to be explicitly considered are listed in Table 2.

Expressions accounting for the contribution of anisotropic hyperfine coupling to $k_{\mathrm{r}}$ have been given by Hayashi and Nagakura ${ }^{13}$ for model radical pairs with one proton spin on each radical. In our systems, the radicals carry one (PXZ radical cation) or two (diquat cation radical) ${ }^{14} \mathrm{~N}$ spins with $I_{\mathrm{N}}=1$. The anisotropic hyperfine interaction in these radicals is by far dominated by these nuclei. Therefore, we derived appropriate expressions accounting for this situation. Starting from the general equation for a radical pair with two nuclear spins of $I$ $=1$ in one radical, that is, the diquat cation radical

$$
\begin{aligned}
& k_{\mathrm{r}, \mathrm{a}}= \\
& \quad \frac{4}{9} \frac{\gamma_{\mathrm{e}}{ }^{2} \tau_{\mathrm{c}}}{\left(1+\omega_{0}{ }^{2} \tau_{\mathrm{c}}{ }^{2}\right)} \sum_{k, l, m, n} \overline{\left|\left\langle T_{+}, k, l\left|\mathbf{S}_{1}\left(\mathbf{t}_{\mathrm{N} 1} \mathbf{I}_{\mathrm{N} 1}+\mathbf{t}_{\mathrm{N} 2} \mathbf{I}_{\mathrm{N} 2}\right)\right| T_{o}, m, n\right\rangle\right|^{2}}
\end{aligned}
$$

where the indices $k, l, m$, and $n$ run over the $(1,0,-1)$ hyperfine states of the two nitrogen nuclei, we arrive at

$$
k_{\mathrm{r}, \mathrm{a}}=\frac{383}{405}(\Delta A)^{2} \frac{\gamma_{\mathrm{e}}{ }^{2} \tau_{\mathrm{c}}}{1+\omega_{0}{ }^{2} \tau_{\mathrm{c}}{ }^{2}}
$$

In eq 9 a factor of 2 is included, taking into account that the transitions between $\mathrm{T}_{+}$(or $\mathrm{T}_{-}$) and $\mathrm{T}_{o}$ as well as $\mathrm{S}$ contribute to $k_{\mathrm{r}}$ and that their probabilities are equal. In eq $10, \gamma_{\mathrm{e}}$ denotes the gyromagnetic ratio of the electron, $\tau_{\mathrm{c}}$ is the orientational correlation time, and $\omega_{0}$ is the angular Larmor frequency. The symbols $\mathbf{S}_{1}, \mathbf{I}_{\mathrm{N} 1}$, and $\mathbf{I}_{\mathrm{N} 2}$ denote the vector operators of electron spin and nuclear spins, respectively, on the diquat radical; $\mathbf{t}_{\mathrm{N} 1}$ and $\mathbf{t}_{\mathrm{N} 2}$ are the anisotropic hyperfine tensors of the two nitrogen nuclei. We assume axial symmetry and hence may neglect that their main axes $x$ and $y$ are rotated with respect to each other around the common $z$ axis, the one perpendicular to the aromatic plane. Thus

$$
\mathbf{t}_{\mathrm{N} 1}=\mathbf{t}_{\mathrm{N} 2}, t_{\mathrm{N}, x x}=t_{\mathrm{N}, y y}=A_{\perp}, t_{\mathrm{N}, z z}=A_{\|}
$$

For axially symmetric tensors a single quantity $\Delta A$ is defined as

$$
\Delta A \equiv A_{\|}-A_{\perp}
$$

is sufficient to define the anisotropy.

For the combined action of anisotropic hyperfine coupling and g-tensor anisotropy as taking place in the PXZ cation radical, we take into account the anisotropic hyperfine coupling of one nitrogen nucleus. The analogue of eq 9 is

$$
k_{\mathrm{r}, \mathrm{ag}}=\frac{4}{3} \frac{\gamma_{\mathrm{e}}{ }^{2} \tau_{\mathrm{c}}}{\left(1+\omega_{0}{ }^{2} \tau_{\mathrm{c}}{ }^{2}\right)} \sum_{k, l} \overline{\left|\left\langle T_{+}, k\left|\mathbf{S}_{1}\left(\mathbf{t}_{\mathrm{N}} \mathbf{I}_{\mathrm{N}}+\mathbf{g}^{\prime} \mathbf{B}_{0} / g_{e}\right)\right| T_{o}, l\right\rangle\right|^{2}}
$$

The symbol $\mathbf{g}^{\prime}$ denotes the anisotropic part of the $g$ tensor. We assume that it is axially symmetric and that its main axis coincides with the axis of the anisotropic hyperfine tensor $\mathbf{t}_{\mathrm{N}}$. As for the anisotropic hyperfine tensor, the effect of the axially symmetric anisotropic $g$ tensor can be expressed by a single quantity:

$$
\Delta g^{\prime}=\Delta g \equiv g_{\|}-g_{\perp}
$$

It should be noted that the effects of ahfi and gta are not simply additive: there is an interference term. Evaluating the matrix elements in eq 13 , we obtain

$$
\begin{array}{r}
k_{\mathrm{r}, \mathrm{ag}}=\frac{4}{27}(\Delta A)^{2} \frac{\gamma_{\mathrm{e}}{ }^{2} \tau_{\mathrm{c}}}{1+\omega_{0}{ }^{2} \tau_{\mathrm{c}}{ }^{2}}-\frac{1}{90}(\Delta g \Delta A) \frac{\gamma_{\mathrm{e}} \omega_{0} \tau_{\mathrm{c}}}{1+\omega_{0}{ }^{2} \tau_{\mathrm{c}}{ }^{2}}+\frac{1}{60} \\
(\Delta g)^{2} \frac{\omega_{0}{ }^{2} \tau_{\mathrm{c}}}{1+\omega_{0}{ }^{2} \tau_{\mathrm{c}}{ }^{2}}
\end{array}
$$

An expression for the longitudinal and transversal relaxation times $T_{1}$ and $T_{2}$ of a radical due to spin-rotational relaxation has been derived by Atkins and Kivelson. ${ }^{33,34}$ If one radical in the radical pair relaxes due to spin rotational interaction, then the contribution to $k_{\mathrm{r}}$ in the radical pair is given by

$$
k_{\mathrm{r}, \mathrm{sri}}=\frac{1}{2 T_{1, \mathrm{sri}}}=\frac{1}{2 T_{2, \mathrm{sri}}}=\frac{1}{18} \frac{\overline{\delta g^{2}}}{\tau_{\mathrm{c}}}
$$


where $\overline{\delta g^{2}}$ is given by

$$
\overline{\delta g^{2}}=\sum_{i=x, y, z}\left(g_{i i}-g_{\mathrm{e}}\right)^{2}
$$

with $g_{i i}$ being the main values of the diagonal $g$ tensor and $g_{\mathrm{e}}$ being the $g$ factor of the free electron. Assuming the $g$ tensor to be axially symmetric, and spin-orbit coupling effects at the heavy atom center in the aromatic ring to contribute only to the components in the ring plane, we have

$$
g_{\|}=g_{\mathrm{e}} \quad \text { and } g_{\perp}=\left(3 g-g_{\mathrm{e}}\right) / 2
$$

where $g$ is the isotropic average of the $g$ factor. Hence

$$
\overline{\delta g^{2}}=\frac{9}{2}\left(g-g_{\mathrm{e}}\right)^{2}
$$

The role of electron spin dipolar interaction on electron spin relaxation in the confined space of a micellar supercage, where the stochastic modulation of the interaction is due to the translational diffusion of the two radicals in the micelle, has been quantitatively analyzed by Steiner and $\mathrm{Wu}^{35}$ using Monte Carlo simulations. If $r_{0}$ denotes the contact distance of the two radicals and $r_{\mathrm{M}}$ is the radius of the micelle, then the result can be cast into the following phenomenological form

$$
\begin{aligned}
& k_{\mathrm{r} \text {,esdi }}=k_{T} \pm_{\rightarrow T_{0} \text {,esdi }}=\frac{3}{10} \hbar^{2} \gamma_{\mathrm{e}}{ }^{4} r_{0}{ }^{-3} r_{\mathrm{M}}{ }^{-3}\left(\frac{a_{1} \tau_{1}}{1+\omega_{0}{ }^{2} \tau_{1}^{2}}+\right. \\
& \left.\frac{a_{2} \tau_{2}}{1+\omega_{0}^{2} \tau_{2}^{2}}\right)
\end{aligned}
$$

with $a_{1}=0.6, a_{2}=0.4, \tau_{1}=1.10 \times 10^{-16} \mathrm{~cm}^{2} \mathrm{~s}^{-1} D^{-1}$, and $\tau_{2}$ $=7.70 \times 10^{-16} \mathrm{~cm}^{2} \mathrm{~s}^{-1} D^{-1}$. The symbol $D$ denotes the sum of the diffusion coefficients of the two radicals. Later these results were corroborated analytically by Isakov et al. ${ }^{36}$ The situation in the present linked radical pairs is very similar to the situation in a micelle. The conformational changes of the linkages modulate the distance of the two radical moieties between a separation $r_{0}$ of closest approach and a length $r_{M}$ of most distant separation. The conformational chain dynamics can be approximated by a diffusional process of the radical moieties with a phenomenological diffusion constant $D$ (for a similar treatment of energy transfer, cf. refs 37 and 38).

To assess the parameters of the various mechanistic contributions from the observed experimental data on $k_{\mathrm{r}}$, we start by comparing the high-field values for the DQ-PSZ pair with the results for the EPR line width of the Me-PSZ cation radical (cf. Table 1). This line width exceeds the values for Me-POZ and Me-PTZ by about $0.25 \mathrm{G}$. This suggests assigning the difference to the effect of spin-rotational interaction. ${ }^{39} \mathrm{~A}$ Lorentzian width contribution of $0.25 \mathrm{G}$ corresponds to a value of $1 / T_{2}$ of $7.4 \times 10^{6} \mathrm{~s}^{-1}$. If we assume that the same mechanism is operative in the PSZ moiety of the DQ-PSZ radical pair, then a $k_{\mathrm{r} \text {,sri }}$ value of $3.7 \times 10^{6} \mathrm{~s}^{-1}$ would be expected on the basis of eq 16. Allowing for the uncertainty of the EPR line width, a

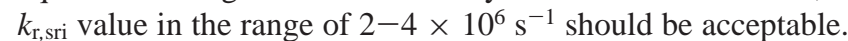
This is in good agreement with the limiting high-field value for the DQ-PSZ radical pair of about $3.5-4 \times 10^{6} \mathrm{~s}^{-1}$, which thus may be largely assigned to the sri mechanism (for a discussion of the weak field dependence of $k_{\mathrm{r}}$ at high fields, cf. below). A contribution of $k_{\mathrm{T}}$, however, on the order of $1 \times 10^{6}$ $\mathrm{s}^{-1}$ cannot be ruled out.
From the isotropic $g$ factor of 2.015 for the Me-PSZ radical cation, we obtain $\overline{\delta g^{2}}=7.26 \times 10^{-4}$. Assuming $k_{\mathrm{r}, \mathrm{sri}}=3.5 \times$ $10^{6}$ and using eq 16 yields an orientational correlation time $\tau_{\mathrm{c}}$ of 11.5 ps. We may convert this value into an effective hydrodynamic radius $r$ of the linked PSZ radical by resorting to the Debye equation ${ }^{40}$

$$
\tau_{\mathrm{c}}=\frac{4 \pi \eta r^{3}}{3 k T}=10^{-12} \frac{\eta}{c P} \frac{r^{3}}{\AA^{3}} s
$$

On the basis of a value of $\eta=0.62 \mathrm{cP}$ for the viscosity of nitromethane at room temperature, we obtain $r=2.65 \AA$, which is a reasonable order of magnitude for the size of the donor and the acceptor moieties. Alternatively, this result, which is based on the bulk viscosity of the solvent, indicates that the rotational mobility of the linked PSZ species does not seem to be severely impeded by the tetramethylene linker.

We now turn to the interpretation of the low-field limits of $k_{\mathrm{r}}$ for which, in the case with the POZ-moiety, only the ahfi mechanism and the esdi mechanism can be invoked. The anisotropic hyperfine coupling of the nitrogen nucleus may be estimated by analogy with the stable TEMPO radical. ${ }^{41}$ Here the isotropic hyperfine coupling constant of nitrogen is $A_{\mathrm{N}}=$ $16.6 \mathrm{G}$ and $\Delta A_{\mathrm{N}}=29.5 \mathrm{G}$. We assume that the ratio of 1.77 between $\Delta A_{\mathrm{N}}$ and $A_{\mathrm{N}}$ can be also applied to other nitrogencentered radicals. For the DQ radical cation, the two isotropic nitrogen couplings are $A_{\mathrm{N}}=4.0 \mathrm{G},{ }^{42}$ leading to an estimation of $\Delta A_{\mathrm{N}}=7.1 \mathrm{G}$, and for POZ the nitrogen coupling is taken as $A_{\mathrm{N}}=9.2 \mathrm{G}$ as listed in Table 1 , leading to an estimated $\Delta A_{\mathrm{N}}$ $=15.9 \mathrm{G}$. Employing these values together with the value of the orientational correlation time $\tau_{\mathrm{c}}$ obtained above from the contribution of the spin-rotational mechanism, we obtain $k_{\mathrm{r}, \mathrm{a}} \mathrm{POZ}$ $=3.0 \times 10^{5} \mathrm{~s}^{-1}$ at $10 \mathrm{mT}$, the lowest field, for which the experimental value of $k_{\mathrm{r}}$ has been unambiguously determined. The experimental value of $5.4 \times 10^{6} \mathrm{~s}^{-1}$ is more than 10 times larger than the theoretical value from the ahfi mechanism. Also, as a consequence of the used correlation time of $11.5 \mathrm{ps}, k_{\mathrm{r}, \mathrm{a}} \mathrm{POZ}$ is constant up to about $100 \mathrm{mT}$, which is at variance with the experimental behavior.

Thus, it is clear that the ahfi mechanism cannot account for the behavior of $k_{\mathrm{r}}$ in the low field region up to about $100 \mathrm{mT}$. Electron spin dipolar interaction is a promising candidate to fill this gap. To apply eq 20, we need to fix the parameters $r_{0}$, the distance of closest approach of the two radicals, $r_{\mathrm{M}}$, the maximum distance, and $D$, the effective relative diffusion constant of the two radicals. For $r_{0}$ we chose the value of $2 r$, that is, twice the value of the hydrodynamic radius of the individual radicals; for $r_{\mathrm{M}}$ a value of $20 \AA$ can be estimated from a molecular model. It has already been pointed out that the conformational chain dynamics of the linkers, which determines the modulation of the distance between the two radicals, can be simulated by a diffusion model. It should, however, not be expected that this diffusion is as fast as that for unbound molecules in homogeneous solution. Nevertheless, we may estimate an upper limit of the corresponding diffusion constant using the Einstein-Stokes relation

$$
D=D_{\mathrm{DQ}}+D_{\mathrm{PXZ}}=\frac{2 k T}{6 \pi r \eta}=4.37 \times 10^{-5}\left(\frac{r \eta}{\AA \mathrm{\AA PP}}\right)^{-1} \frac{\mathrm{cm}^{2}}{\mathrm{~s}}
$$

yielding $D=2.3 \times 10^{-5} \mathrm{~cm}^{2} \mathrm{~s}^{-1}$. The expected field dependence of $k_{\mathrm{r} \text {,esdi }}$ obtained with these parameters is shown in Figure 10. It is evident that the $k_{\mathrm{r} \text {,esdi }}$ values obtained for $D>10^{-5} \mathrm{~cm}^{2} \mathrm{~s}^{-1}$ are much too small. However, by decreasing the effective value 


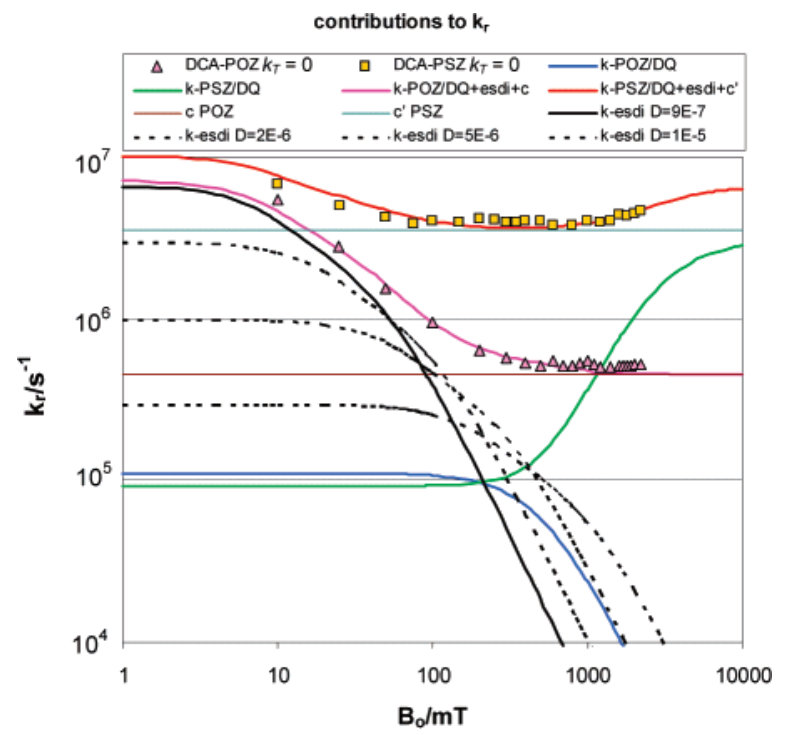

Figure 10. Contributions to $k_{\mathrm{r}}$ in the theoretical simulation of spin relaxation in the radical pairs from DCA-POZ and DCA-PSZ evaluated under the assumption of $k_{\mathrm{T}}=0$ (cf. data points). The full simulations are represented by the curves denoted $k$-POZ/DQ + esdi $+c$ and $k$-PSZ/ $\mathrm{DQ}+$ esdi $+c^{\prime}$, respectively. The contributions from the esdi mechanism ( $k$-esdi $D=\ldots$ ) are given for various values of the effective diffusion constant $D$. The best fit is for $D=9 \times 10^{-7} \mathrm{~cm}^{2} \mathrm{~s}^{-1}$. The curves indicated as $k$-POZ/DQ and $k$-PSZ/DQ, respectively, represent the sum of the contributions of the ahfi and gta mechanisms. The constant values $c$ POZ and $c^{\prime}$ PSZ represent the field-independent contributions to $k_{\mathrm{r}}$. For details of the calculation, cf. text.

of $D$ and keeping all of the other parameters as given, the field dependence of $k_{\text {r,esdi }}$ nicely approaches the observed behavior. For $D=9.0 \times 10^{-7} \mathrm{~cm}^{2} \mathrm{~s}^{-1}$, not only the low-field values for POZ and for PSZ are well-approximated but also the field dependence up to $100 \mathrm{mT}$. It has been noted earlier by Steinberg and co-workers ${ }^{37,38}$ in the theoretical study of energy transfer in linked donor-acceptor systems that the effective relative diffusion constant for two chain ends attached to an oligopeptide linker is much slower than that for the unbound donor and acceptor molecule. Values in the range between $0.9 \times 10^{-7}$ $\mathrm{cm}^{2} \mathrm{~s}^{-1}$ and $6.4 \times 10^{-7} \mathrm{~cm}^{2} \mathrm{~s}^{-1}$ for the diffusion constant were found to appropriately describe the behavior of 4-9 amino acid oligopeptides in ethanol as the solvent. In the light of these findings, the present observation of an effective diffusion constant of $9.0 \times 10^{-7} \mathrm{~cm}^{2} \mathrm{~s}^{-1}$ seems quite reasonable and strongly supports the leading role of the esdi spin relaxation mechanism in linked radical pairs at low fields.

At high fields, the $k_{\mathrm{r}}$ contribution of the esdi mechanism drops below the experimental values, which approach a magneticfield-independent limit. If this limiting value is added as a constant contribution $\left(4.5 \times 10^{5} \mathrm{~s}^{-1}\right.$ in the case of DCA-POZ) to the esdi contribution, then the full field dependence is quite well reproduced. The $k_{\mathrm{r} \text {,esdi }}$ contribution as adapted to the field dependence of the DCA-POZ data yields, at the same time, an excellent description of the field dependence of the DCA-PSZ data if the appropriate constant value of $k_{\mathrm{r}, \mathrm{sri}}$, as discussed above, is added.

Now we consider the role of the ahfi mechanism at higher fields. As can be seen in Figure 10 for the case of DCA-POZ, up to about $75 \mathrm{mT}$ the ahfi contribution is much below the esdi contribution. At $150 \mathrm{mT}$ the order of these two mechanisms is reversed, and at fields above $500 \mathrm{mT}$ the ahfi contribution is about 2 orders of magnitude larger than the esdi contribution. Nevertheless, at all fields the ahfi contribution is much smaller than the observed $k_{\mathrm{r}}$. If it is assumed that the limiting high field value of $k_{\mathrm{r}}$ is constantly contributing at all fields, then in the field region between 50 and $600 \mathrm{mT}$, the overall sum including ahfi results in up to $50 \%$ too high values for $k_{\mathrm{r}}$, indicating an overestimation of the ahfi contribution. A good fit is obtained (cf. Figure 10) if the anisotropy $\triangle A$ is taken only to be a factor of 1.1 times the isotropic hyperfine constant of the nitrogen atoms.

None of the mechanisms discussed so far can account for the limiting high-field value of about $5 \times 10^{5} \mathrm{~s}^{-1}$ in the DCAPOZ case. Although in the case of DCA-PSZ the magneticfield-independent contribution to $k_{\mathrm{r}}$ is explicitly accounted for by the spin-rotational mechanism, this mechanism has only a negligible contribution (about $10^{3} \mathrm{~s}^{-1}$ ) to the field-independent part of $k_{\mathrm{r}}$ in the case of POZ. At present, we cannot assign an explicit mechanism for it. After consideration of all rotational and translational contributions to the stochastic modulation of magnetic interactions as possible sources of spin relaxation, only local inner vibrational motions remain as have been invoked for contributions to radical spin relaxation occasionally. ${ }^{43}$ In the case of DCA-PTZ, the high field limit of $k_{\mathrm{r}}$ is similar to POZ. However, here the theoretical contribution of the sri mechanism $\left(3.5 \times 10^{5} \mathrm{~s}^{-1}\right)$ is considerable and accounts for the largest part if not all of $k_{\mathrm{r}}$.

In the case of the DCA-PSZ radical pair, a slight but significant decrease of $k_{\mathrm{r}}$ at high fields is observed. Similar highfield reversions of magnetic-field effects with radical pairs have been observed by Hayashi and co-workers. ${ }^{15,44,45}$ In principle, two $g$-tensor-dependent mechanistic explanations can account for such observations:

i. the Zeeman mechanism (or " $\Delta g$ mechanism"), ${ }^{46}$ which is due to a magnetic-field-dependent enhancement of the coherent $\mathrm{T}_{0}-\mathrm{S}$ process, due to different $g$ factors of the two radicals and

ii. the gta mechanism, which is due to a magnetic-fielddependent enhancement of spin relaxation in the individual radicals.

The former mechanism only becomes apparent if the frequency of $\mathrm{T}_{0}-\mathrm{S}$ mixing due to the coherent action of isotropic hyperfine interaction is not sufficient to establish $\mathrm{T}_{0}-\mathrm{S}$ spin equilibrium between two radical pair encounters. The latter mechanism is slower than or comparable with the frequency of reencounters. If the effect of a magnetic field is observed through a time-integrated observable, for example, as the radical escape yield in case of unlinked radical pairs, then a decision as to whether mechanism i or ii is operating may be found by using a paramagnetic quencher, which, at not too high of a concentration, can only affect the gta relaxation mechanism. ${ }^{15}$ The TEMPO effect on $\tau_{\text {slow }}$ shown in Figure 7 is a clear indication that it is the gta mechanism that is working here; what we see is that not only the value of $\tau_{\text {slow }}$ in general becomes shorter by the addition of TEMPO but also that the high-field reversion of the magnetic-field effect disappears at higher TEMPO concentration.

Another criterion against the Zeeman mechanism as an explanation of the observed high-field reversion of the magneticfield effect in the DCA-PSZ system is the observed validity of the kinetic model. If this model is correct, then effects according to the Zeeman mechanism cannot show up in the kinetics because the model assumes establishment of the $\mathrm{T}_{0}-\mathrm{S}$ equilibrium at any stage of the recombination. The fact that the model is indeed correct is borne out in the amplitude ratio of the slow and fast decay components, which is not a free parameter in the fitting of the kinetic curves but a function of the adopted rate constants (cf. eq 4). 
The effect of the gta relaxation mechanism can be assessed by eq 15 . At high fields, the quadratic term in $\Delta g$ dominates. It leads to a contribution to $k_{\mathrm{r}}$, which increases quadratically with the magnetic field $B_{0}$. At high fields, however, it approaches a constant limit. The half field value $B_{1 / 2, \text { gta }}$ for the saturation occurs at

$$
B_{1 / 2, \mathrm{gta}}=\frac{1}{\gamma_{\mathrm{e}} \tau_{\mathrm{c}, \mathrm{gta}}}
$$

For a simulation (cf. Figures 6 and 10), all parameters except for $\tau_{\mathrm{c} \text {,gta }}$ are known. An optimum fit is achieved for $\tau_{\mathrm{c}, \mathrm{gta}} \approx 2$ ps. This value is about 1 order of magnitude shorter than that reasonably expected for rotational tumbling (cf. eq 21) of the PSZ radical moiety. A stochastic motion modulating the $g$ tensor might be sought in a modulation of the butterfly-like central ring deformation in the PSZ molecule. Because of the nonplanar hybridization of the methylated ring nitrogen, ${ }^{47}$ this species (and its radical) is bent along the axis connecting the two central heteroatoms. By Raman spectroscopy as well as by DFT calculations, several thermally easily excitable vibrational modes of the central ring deformation have been assessed in the range of $50-500 \mathrm{~cm}^{-1} .^{48}$ Variations of vibrational excitation of these modes will affect the $g$ tensor. Vibrational correlation times on the order of some picoseconds (corresponding to vibrational linewidths of several $\mathrm{cm}^{-1}$ ) appear reasonable and could account for the observation of a correlation time $\tau_{\mathrm{c}, \mathrm{gta}}$ as short as that observed.

Paramagnetic Quenching of the Magnetic-Field Effect by TEMPO. Several studies of the effects of paramagnetic species on spin chemical magnetic-field effects have been reported in the literature. Among the species that have figured prominently as paramagnetic perturbers are $\mathrm{Ln}^{3+}$ ions ${ }^{49-52}$ and transitionmetal ions, ${ }^{49,53}$ nitroxyl radicals (in particular TEMPO), ${ }^{54-58}$ and molecular oxygen. ${ }^{59}$ In some cases, the paramagnetic perturber is covalently linked to either the biradical species ${ }^{57,58}$ or to one of the radicals in the radical pair. ${ }^{55}$ In these cases, the total spin system is strongly coupled. It is the situation of a triplet-doublet pair wherein the coupled spin states of a quartet and a doublet that are energetically separated by considerable exchange splitting, are kinetically relevant. In our experiment with unlinked TEMPO, this perturber interacts with the rather weakly coupled radicals of the CS state. Formally, it enhances the transitions between $\mathrm{T}_{ \pm}$and $\mathrm{T}_{0} / \mathrm{S}$, or the transitions between $\mathrm{T}_{0}$ and $\mathrm{S}$. In a magnetic field, the latter occur on a much faster time scale than the former and rather high concentrations of the paramagnetic perturber would have to be used to affect the $\mathrm{T}_{0}$ and $\mathrm{S}$ process. Such enhancements of $\mathrm{T}_{0} / \mathrm{S}$ transitions have been observed with a $10^{-2} \mathrm{M}$ concentration of $\mathrm{Gd}^{3+}$ ions. ${ }^{15}$ With a TEMPO concentration of $0.1 \mathrm{M}$, even in zero field, a noticeable effect was observed on the recombination of a radical pair. ${ }^{51}$ For the low TEMPO concentrations employed in our experiments only the slow $\mathrm{T}_{ \pm} \rightarrow \mathrm{T}_{0} / \mathrm{S}$ relaxation can be affected. This can occur by two mechanisms: electron spin dipolar interaction, or Heisenberg spin exchange. If the latter mechanism operates between typical organic radicals in low viscous solvents, then rate constants of $2-4 \times 10^{9} \mathrm{M}^{-1} \mathrm{~s}^{-1}$; that is, close to diffusion controlled, seem to be typical. ${ }^{60,61}$ The relaxation effect due to the electron spin dipolar interaction between a paramagnetic perturber and a radical can be estimated according to an equation derived by $\mathrm{RaO}^{49,62}$

$$
k_{q-\text { esdi }}=\frac{8 \pi N_{A}}{30 r_{0}{ }^{3}} \gamma_{\mathrm{e}}^{2} \mu^{2} \tau_{\mathrm{c}}\left(1+\frac{3}{1+\omega_{0}{ }^{2} \tau_{\mathrm{c}}{ }^{2}}+\frac{6}{1+4 \omega_{0}{ }^{2} \tau_{\mathrm{c}}{ }^{2}}\right)
$$

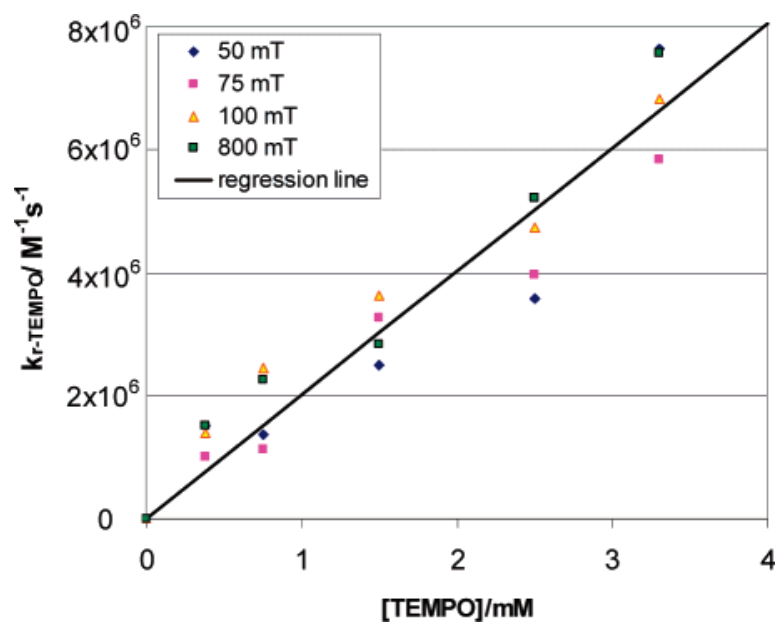

Figure 11. Contribution $k_{\mathrm{r}, \mathrm{TEMPO}}$ to spin relaxation as a function of TEMPO concentration for various magnetic fields.

where $\mu$, the magnetic moment of the perturber in case of TEMPO, is equal to $1.73 \beta$ ( $\beta$ representing Bohr's magneton) and the correlation time is given by

$$
\tau_{\mathrm{c}}=\frac{12 \pi r_{0}^{3}}{k T}
$$

Using a value of $5 \AA$ for $r_{0}$, the distance of closest approach, one obtains $\tau_{\mathrm{c}}=710 \mathrm{ps}$. In this case, eq 24 predicts a limiting, constant value of $k_{q-\text { esdi }}$ at fields above about $50 \mathrm{mT}$. This is the region where the paramagnetic quenching effect has been investigated in our experiments. The saturation value, $2.3 \times$ $10^{8} \mathrm{M}^{-1} \mathrm{~s}^{-1}$, is about 10 times smaller than the value to be expected for the Heisenberg spin exchange mechanism.

From the time regime of the magnetic-field-dependent kinetics where the TEMPO effect is observed, as well as from the low concentrations of TEMPO employed, it is clear that in our experiments this quencher accelerates the $\mathrm{T}_{ \pm} \rightarrow \mathrm{T}_{0}, \mathrm{~S}$ relaxation processes. Denoting this contribution of relaxation by $k_{\mathrm{r}, \mathrm{TEMPO}}$, the total rate constant of relaxation can be decomposed as

$$
k_{\mathrm{r}}=k_{\mathrm{r},[0]}+k_{\mathrm{r}, \mathrm{TEMPO}}
$$

where $k_{\mathrm{r}, 0}$ denotes the relaxation constant in the absence of TEMPO. Substituting eq 24 into eq 5 and using the result $k_{\mathrm{T}}=$ 0 arrived at above, we can determine $k_{\mathrm{r}, \text { TEMPO }}$ as follows:

$$
k_{\mathrm{r}, \mathrm{TEMPO}}=\frac{k_{a}\left(k_{a}-k_{\mathrm{S}} / 2\right)}{2\left(k_{a}-k_{\mathrm{S}} / 4\right)}-k_{\mathrm{r},[0]}
$$

The values of $k_{\text {r,TEMPO }}$ as a function of the concentration of TEMPO obtained for various magnetic fields are plotted in Figure 11.

In the field range from 50 to $800 \mathrm{mT}$ where the values of

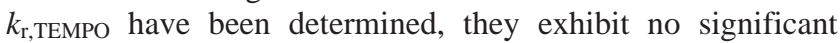
dependence on the magnetic-field strength. The data points are well represented by a linear correlation

$$
k_{\mathrm{r}, \mathrm{TEMPO}}=k_{\mathrm{q}, \mathrm{TEMPO}}[\mathrm{TEMPO}]
$$

with $k_{\mathrm{q}, \mathrm{TEMPO}}=2.0 \times 10^{9} \mathrm{M}^{-1} \mathrm{~s}^{-1}$. This result indicates that the magnetic quenching by TEMPO is close to diffusion controlled and about 10 times faster than that expected for an electron-dipolar relaxation mechanism, but it fits well to the 
typical range observed for Heisenberg spin exchange between organic radicals in low viscous solution (cf. above).

\section{Conclusions}

The conclusions from the analysis of our results may be summarized as follows:

Contributions of direct, spin-forbidden recombination of triplet RPs seem negligible, that is, $k_{\mathrm{T}} \ll k_{\mathrm{r}}(B \rightarrow \infty)$.

Spin-rotational interaction has been explicitly confirmed as being responsible for the saturating limit of the magnetic-field effect in high fields for PSZ. Electron spin dipolar interaction is the dominant relaxation mechanism for the presently investigated linked radical pairs in the field range between 1 and $100 \mathrm{mT}$.

Contributions of anisotropic hyperfine interactions to spin relaxation in the present systems are minor and seem to contribute mainly in the intermediate field region around 200 $\mathrm{mT}$.

The limiting high-field contribution to spin relaxation in the DQ-POZ is not of rotational origin. A vibrational mechanism might be invoked.

The slight increase of $k_{\mathrm{r}}$ at high fields in the case of PSZ is most likely due to the gta mechanism. The extremely short correlation time of about 2 ps for the underlying stochastic modulation of the $g$ tensor is tentatively assigned to a conformational flapping of the ring system about the central $\mathrm{N}-\mathrm{Se}$ axis. The coherent Zeeman mechanism of $\mathrm{T}_{0}-\mathrm{S}$ mixing can definitely be excluded as an explanation of this effect.

Acknowledgment. This work was supported by (C.M.E.) the U.S. Department of Energy, Office of Basic Energy Sciences (DE-FG02-04ER15591). We thank Dr. Karsten Hötzer for experimental support with initial laser experiments and Mr. John Weber for the synthesis of $N$-methylphenoxazine.

\section{References and Notes}

(1) Photoinduced Electron Transfer; Fox, M. A., Chandon, M., Eds.; Elsevier: Amsterdam, 1988; Vols. A-D.

(2) Schanze, K. S.; Walter, K. A. Photoinduced Electron Transfer in Metal-Organic Dyads. In Molecular and Supermolecular Photochemistry Organic and Inorganic Photochemistry; Ramamurthy, V., Schanze, K. S., Eds.; Marcel Dekker: New York, 1998; Vol. 2, p 75.

(3) Kalyanasundaram, K. Photochemistry of Polypyridine and Porphyrin Complexes; Academic Press: San Diego, CA, 1992.

(4) Armaroli, N. Photochem. Photobiol. Sci. 2003, 2, 73.

(5) Scandola, F.; Chiorboli, C.; Indelli, M. T.; Rampi, M. T. Covalently Linked Systems Containing Metal Complexes. In Biological and Artificial Supermolecular Systems; Wiley: Weinheim, 2003; Vol. 3, p 337.

(6) Gust, D.; Moore, T. A.; Moore, A. L. Electron Transfer Chem. 2001, 3, 272.

(7) Gust, D.; Moore, T. A. Porphyrin Handbook 2000, 8, 153.

(8) Klumpp, T.; Linsenmann, M.; Larson, S. L.; Limoges, B. R.; Buerssner, D.; Krissinel, E. B.; Elliott, C. M.; Steiner, U. E. J. Am. Chem. Soc. 1999, 121, 1076.

(9) Danielson, E.; Elliott, C. M.; Merkert, J. W.; Meyer, T. J. J. Am. Chem. Soc. 1987, 109, 2519.

(10) Larson, S. L.; Cooley, L. F.; Elliott, C. M.; Kelley, D. F. J. Am. Chem. Soc. 1992, 114, 9504.

(11) Larson, S. L.; Elliott, C. M.; Kelley, D. F. J. Phys. Chem. 1995, 99, 6530 .

(12) The inclusion of two donor moieties is simply a matter of synthetic expediency (it is easier to prepare the complex containing two donors), and is of no particular consequence in the qualitative behavior of the assemblies.

(13) Hayashi, H.; Nagakura, S. Bull. Chem. Soc. Jpn. 1984, 57, 322.

(14) The rate constant of direct, soc-assisted spin-forbidden recombination is expected to vary as the square of the soc constant, while $g$-tensorrelated effects such as gta and sri should vary linearly.

(15) Wakasa, M.; Sakaguchi, Y.; Hayashi, H. Mol. Phys. 1994, 83, 613.

(16) Rawls, M. T. Ph D. Thesis, Colorado State University, Fort Collins, CO, 2007
(17) Rawls, M. T.; Kollmannsberger, G.; Elliott, C. M.; Steiner, U. E., to be submitted for publication.

(18) Schmehl, R. H.; Ryu, C. K.; Elliott, C. M.; Headford, C. L. E.; Ferrere, S. Adv. Chem. Ser. 1990, 226, 211.

(19) Elliott, C. M.; Freitag, R. A. J. Chem. Soc. Chem. Rev. 1985, 156.

(20) Murray, R. W.; Heineman, W. R.; O’Dom, G. W. Anal. Chem.

1967, 39, 1666.

(21) Linsenmann, M. Ph.D. Dissertation, University of Konstanz, 1997.

(22) Treadway, J. A.; Chen, P.; Rutherford, T. J.; Keene, F. R.; Meyer,

T. J. J. Phys. Chem. A 1997, 101, 6824.

(23) The time constants shown in Figure 6 were taken from a biexponential fit with no restraints as imposed by the kinetic model (cf. eqs 4 and 5). If such restraints are employed, then the data points in the region between 100 and $400 \mathrm{mT}$ are less scattered and show a rather broad plateau around $t_{\text {slow }}=280 \mathrm{~ns}$. At higher fields, both fitting methods yield identical results.

(24) The modest difference between the $k_{a}$ value reported herein and those in ref 8 is likely due to trace oxygen impurities present in the samples considered in the earlier study.

(25) Strictly speaking, when the Zeeman splitting in the external field drops below the isotropic hyperfine coupling, it is no longer appropriate for a substate-resolved kinetic description to use the high-field basis of triplet substates that are oriented in the laboratory frame. In a classical kinetic approximation, one should rather use the low-field eigenstates of the spin Hamiltonian, which are of mixed singlet and triplet character and accordingly differ in their reactivity towards the singlet recombination channel in correspondence with their singlet character. However, within the context of the present work, relaxation between these states is fast enough so that they can be essentially considered as a single kinetic species.

(26) Weller, A.; Nolting, F.; Staerk, H. Chem. Phys. Lett. 1983, 96, 24.

(27) Steiner, U. E.; Wolff, H.-J. Magnetic Field Effects in Photochemistry. In Photochemistry and Photophysics; Rabek, J. F., Ed.; CRC Press Inc.: Boca Raton, FL, 1991; Vol. IV, p 1.

(28) The coefficient $c_{a}$ is not very sensitive to variations in $k_{\mathrm{r}}$ and $k_{\mathrm{T}}$ compatible with a given $k_{a}$, nor is the phenomenological rate constant $k_{b}$.

(29) Steiner, U. E.; Winter, G. Chem. Phys. Lett. 1978, 55, 364.

(30) Carrington, A.; McLachlan, A. D. Introduction to Magnetic Resonance with Applications to Chemistry and Chemical Physics; Chapman and Hall: London, 1979.

(31) Moore, C. E. Atomic Energy Levels; Circular of the National Bureau of Standards No. 467 (U.S.); Vol. I (1949), Vol. II (1952), Vol. III (1953).

(32) Fraga, S.; Malli, G. Many Electron Systems Properties and Interactions; Saunders: Philadelphia, PA, 1968.

(33) Atkins, P. W.; Kivelson, D. J. Chem. Phys. 1966, 44, 169

(34) Steiner, U. E.; Serebrennikov, Y. A. J. Chem. Phys. 1994, 100, 7503

(35) Steiner, U. E.; Wu, J. Q. Chem. Phys. 1992, 162, 53.

(36) Isakov, S. V.; Lukzen, N. N ; Morozov, V. A.; Sagdeev, R. Z. Chem. Phys. 1995, 199, 119

(37) Haas, E.; Katchalski-Katzi, E.; Steinberg, I. Z. Biopolymers 1978, $17,11$.

(38) Katchalski-Katzir, E.; Haas, E.; Steinberg, I. Z. Annu. Rev. N.Y. Acad. Sci. 1981, 366, 44.

(39) At X-band, the line-width contribution of the gta mechanism can be estimated to be about 1 order of magnitude smaller.

(40) Debye, P. Polar Molecules; Dover Publicatons: New York, 1945.

(41) Ottaviani, M. F.; Baglioni, P.; Martini, G. J. Phys. Chem. 1983, $87,3146$.

(42) Rieger, A. L.; Rieger, P. H. J. Phys. Chem. 1984, 88, 5845.

(43) Yong, L.; Harbridge, J.; Quine, R. W.; Rinard, G. A.; Eaton, S. S.; Eaton, G. R.; Mailer, C.; Barth, E.; Halpern, H. J. J. Magn. Reson. 2001, $152,156$.

(44) Wakasa, M.; Hayashi, H.; Mikami, Y.; Takada, T. J. Phys. Chem. 1995, 99, 13181

(45) Wakasa, M.; Igarashi, M.; Sakaguchi, Y.; Hayashi, H. Chem. Lett. 1994, 1991.

(46) In this term, $\Delta g$ stands for the difference of $g$ factors of the two radicals. Because we are already using the symbol $\Delta g$ in a different meaning, that is, as a designation of the $g$-tensor anisotropy, here we will avoid the term " $\Delta g$ mechanism".

(47) Clarke, D.; Gilbert, B. C.; Hanson, P.; Kirk, C. M. J. Chem. Soc., Perkin Trans. 2 1978, 1103.

(48) Pan, D.; Phillips, D. L. J. Phys. Chem. A 1999, 103, 4737.

(49) Kita, Y.; Tanimoto, A.; Itoh, M.; Okazaki, M.; Nakagaki, R. Chem. Phys. Lett. 1990, 165184.

(50) Sakaguchi, Y.; Hayashi, H. Chem. Phys. Lett. 1984, 106, 420.

(51) Turro, N. J.; Lei, X.; Gould, I. R.; Zimmt, M. B. Chem. Phys. Lett. $1985,120,397$.

(52) Basu, S.; Nath, D.; Chowdhury, M. J. Lumin. 1988, 40\&41, 252.

(53) Sakaguchi, Y.; Hayashi, H. Chem. Phys. 1992, 162, 119.

(54) Chen, J.; Mori, Y.; Sakaguchi, Y.; Hayashi, H. Mol. Phys. 2002, $100,1355$. 
(55) Mori, Y.; Sakaguchi, Y.; Hayashi, H. J. Phys. Chem. A 2000, 104, 4869.

(56) Step, E. N ; Buchachenko, A. L.; Turro, N. J. J. Am. Chem. Soc. 1994, 116, 5462.

(57) Mori, Y.; Sakaguchi, Y.; Hayashi, H. J. Phys. Chem. A 2002, 106, 4453.

(58) Mori, Y.; Sakaguchi, Y.; Hayashi, H. Chem. Phys. Lett. 1998, 286,
(59) Levin, P. P.; Kuzmin, V. A. Chem. Phys. 1992, 162, 79.

(60) Eastman, M. P.; Kooser, R. G.; Das, M. R.; Freed, J. H. J. Chem. Phys. 1969, 11, 2690.

(61) Eastman, M. P.; Bruno, G. V.; Freed, J. H. J. Chem. Phys. 1970, $52,2511$.

(62) Hyde, H. S.; Swartz, H. M.; Antholine, W. E. The Spin-ProbeSpin-Label Method. In Spin Labeling; Berliner, L. J., Ed.; Academic Press: New York, 1979; Vol. 2, p 71. 